\title{
Outgoing wave conditions in photonic crystals and transmission properties at interfaces
}

\author{
A. Lamacz, B. Schweizer*
}

December 19, 2016

\begin{abstract}
We analyze the propagation of waves in unbounded photonic crystals. Waves are described by a Helmholtz equation with $x$-dependent coefficients, the scattering problem must be completed with a radiation condition at infinity. We develop an outgoing wave condition with the help of a Bloch wave expansion. Our radiation condition admits a uniqueness result, formulated in terms of the Bloch measure of solutions. We use the new radiation condition to analyze the transmission problem where, at fixed frequency, a wave hits the interface between free space and a photonic crystal. We show that the vertical wave number of the incident wave is a conserved quantity. Together with the frequency condition for the transmitted wave, this condition leads (for appropriate photonic crystals) to the effect of negative refraction at the interface.
\end{abstract}

Keywords: Helmholtz equation, radiation, waveguide, Bloch analysis, outgoing wave condition, photonic crystal, transmission problem, negative refraction

MSC: 35Q60, 35P25, 35B27

\section{Introduction}

Photonic crystals are optical devices that allow to mold the propagation properties of light. They usually have a periodic structure and are operated with light at a fixed frequency $\omega$. Due to their spectral properties (band gap structure), light of certain frequencies can travel in the photonic crystal, but, at other frequencies, the crystal is opaque. A large body of literature is available on this aspect of photonic crystals. Most contributions study a periodic medium, possibly with a compactly supported perturbation of the periodic structure. In contrast, we are interested in the interface between a photonic crystal and free space.

${ }^{*}$ Technische Universität Dortmund, Fakultät für Mathematik, Vogelpothsweg 87, D-44227 Dortmund, Germany. agnes.lamacz@tu-dortmund.de, ben.schweizer@tu-dortmund.de 
An interesting effect of such an interface is negative refraction. A recent discussion in the physical literature concerns the following question: Is negative refraction always the result of a negative index of the photonic crystal, or can negative refraction also occur at the interface between air and a photonic crystal with positive index? Our mathematical results confirm the latter: The conservation of the transversal wave number can lead to negative refraction between two materials with positive index, as suggested in [27].

In mathematical terms, the light intensity is determined by the Helmholtz equation

$$
-\nabla \cdot(a(x) \nabla u(x))=\omega^{2} u(x),
$$

which must be solved for $u$ in a domain $\Omega, u=u(x), x=\left(x_{1}, x_{2}\right) \in \Omega$. Here, we restrict our analysis to an unbounded rectangle $\Omega:=\mathbb{R} \times(0, h) \subset \mathbb{R}^{2}$, but note that our methods can also be used in higher dimension, e.g. for $\Omega:=\mathbb{R} \times\left(0, h_{2}\right) \times\left(0, h_{3}\right) \subset \mathbb{R}^{3}$. In (1.1), $\omega>0$ is a prescribed frequency and $a=a(x)$ is the inverse permittivity of the medium. In an $x_{3}$-independent geometry and with polarized light, the time-harmonic Maxwell's equations reduce to (1.1) and $u=u(x)$ is the out-of-plane component of the magnetic field.

The coefficient $a=a(x)$ describes the medium. We assume that the right half space $\left\{x=\left(x_{1}, x_{2}\right) \in \Omega \mid x_{1}>0\right\}$ is occupied by a periodic photonic crystal with periodicity length $\varepsilon>0$. Using the unit cube $Y=(0,1)^{2}$ and the scaled cube $Y_{\varepsilon}=\varepsilon Y=(0, \varepsilon)^{2}$, we therefore assume that the coefficient $a=a^{\varepsilon}$ is $Y_{\varepsilon}$-periodic for $x_{1}>0$. We make the assumption that an integer number $K$ of cells fits vertically in the domain, i.e. $K=$ $h / \varepsilon \in \mathbb{N}$. On the left half space $\left\{x=\left(x_{1}, x_{2}\right) \in \Omega \mid x_{1}<0\right\}$, we set $a=a^{\varepsilon} \equiv 1$. With $a=a^{\varepsilon}$ and $\omega$ given, problem (1.1) is an equation for $u$, but it must be accompanied by boundary conditions.

We impose periodic boundary conditions in the vertical direction, i.e. we identify the lower boundary $\left\{x=\left(x_{1}, x_{2}\right) \mid x_{2}=0\right\}$ with the upper boundary $\left\{x=\left(x_{1}, x_{2}\right) \mid x_{2}=h\right\}$. In order to analyze scattering properties of the interface, we assume that the interface is lit by a planar wave. We consider, for a fixed wave-vector $k \in \mathbb{R}^{2}$, the incident wave

$$
U_{\text {inc }}(x)=e^{2 \pi i k \cdot x / \varepsilon} \text {. }
$$

To guarantee that $U_{\text {inc }}$ is a solution to (1.1) on the left, we assume $\omega^{2}=4 \pi^{2}|k|^{2} / \varepsilon^{2}$. Since the Helmholtz-equation models a time-harmonic situation, we should think here of a solution of the wave equation in the form $\hat{U}_{\text {inc }}(x, t)=U_{\text {inc }}(x) e^{-i \omega t}=\exp (i[2 \pi k$. $x / \varepsilon-\omega t])$. We always consider $k_{1}>0$ such that $U_{\text {inc }}$ represents a right-going wave. In addition, we assume that the incident wave respects the periodicity condition in vertical direction, i.e. $e^{2 \pi i k_{2} h / \varepsilon}=1$ or, equivalently, $k_{2} h \in \varepsilon \mathbb{Z}$.

With the incident wave $U_{\text {inc }}$ at hand we can now describe - at least formally - the boundary conditions for solutions $u$ of (1.1) as $x_{1} \rightarrow \pm \infty$. We seek for $u$ such that (i) $u$ satisfies an outgoing wave condition as $x_{1} \rightarrow \infty$ and (ii) $u-U_{\text {inc }}$ satisfies an outgoing wave condition for $x_{1} \rightarrow-\infty$. This leads us to our first question:

Question 1: How can we prescribe radiation conditions in periodic media?

The answer to Question 1 is intricate and requires a detailed study. We will use Bloch expansions and Bloch projections to formulate our new outgoing wave condition in 


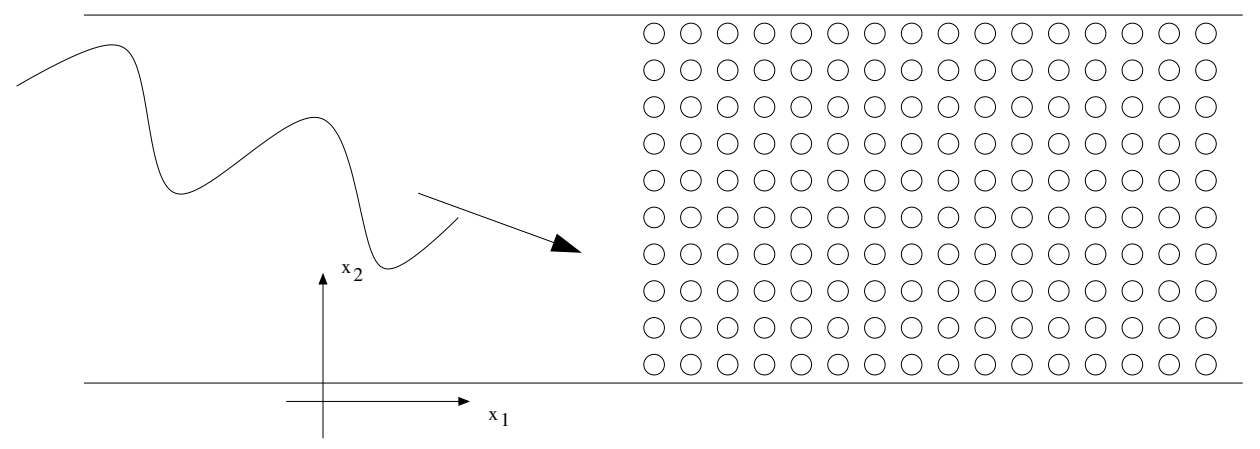

Figure 1: The geometry of the transmission problem for $K=10$ (number of cells in vertical direction). An incoming wave hits the boundary of a photonic crystal. We are interested in the waves that are generated in the photonic crystal.

Definition 3.3. In order to motivate our choice, we sketch some background in the next subsection.

Once we have a precise formulation of the scattering problem, we can turn to the application: What can be said about the transmission problem? When an incident wave $U_{\text {inc }}$ lights the interface, it creates waves inside the photonic crystal. These waves are described by $u$ on $\left\{x_{1}>0\right\}$, our aim is to characterize $u$. Performing a Bloch expansion, we write $u$ as a superposition of Bloch waves. In this superposition, we expect that there appear only waves that satisfy two requirements: (a) the Bloch frequency coincides with the frequency $\omega$. (b) the vertical wave number of the Bloch wave is $k_{2}$ ("conservation of the vertical wave number").

Question 2: Let $u$ be the solution of the transmission problem for the incoming wave $U_{\text {inc }}$. Does the Bloch expansion of $u$ on the right respect the frequency condition and the conservation of the vertical wave number?

A positive answer to Question 2 provides information on the negative refraction phenomenon. The requirements (a) and (b) are used in [27] to explain negative refraction without referring to a negative index material: Denoting the $m$-th Bloch eigenvalue for the wave-vector $j \in Z:=[0,1]^{2}$ as $\mu_{m}(j)$, the photonic crystal can have the property that the three conditions (a) $\mu_{0}(j)=\omega^{2}$, (b) $j_{2}=k_{2}$, and the additional condition (c) $e_{1} \cdot \nabla_{j} \mu_{0}(j)>0$ (the group velocity has a positive $x_{1}$-component), determine $j$ uniquely. For an appropriately chosen field $a$, an appropriate frequency $\omega$ and an appropriate incoming wave vector $k$, we have the following situation: $e_{2} \cdot k=k_{2}$ is negative, but the solution $j$ satisfies $e_{2} \cdot \nabla_{j} \mu_{0}(j)>0$. This means that a light beam that hits the interface from above $\left(k_{2}\right.$ negative in free space implies that the vertical group velocity is negative) produces a light beam in the photonic crystal that is directed towards the top (vertical group velocity is positive, $e_{2} \cdot \nabla_{j} \mu_{0}(j)>0$ ). With this mechanism, the conditions (a)-(b) can lead to negative refraction. This is outlined in [27], where a specific photonic crystal is described and the negative refraction effect is supported by numerical results. We note that a quite different interpretation is given in [12]. 
We will answer the above Questions 1 and 2. The precise answers are more complex than one might expect at first sight (we sketch some of the principal difficulties in the next two subsections). We show that our outgoing wave condition of Definition 3.3 is reasonable by proving a uniqueness result: Theorem 1.3 yields, in a weak sense, the uniqueness of solutions in terms of the Bloch measure. Question 2 is answered with Theorem 1.5. If $u$ is a solution that satisfies the outgoing wave condition, then the corresponding Bloch measure is concentrated in those frequencies that respect (a)-(b). The mathematical description of our results is given in Section 1.3 .

\subsection{Outgoing wave conditions}

Although we use our results to analyze negative refraction, the core of our mathematical theory is more general: We develop an outgoing wave condition for the Helmholtz equation in a periodic medium. In this section, we sketch some background concerning radiation conditions, mainly in free space. Our aim is to demonstrate the importance of radiation conditions, to show the intimate link between radiation conditions and uniqueness results, and to motivate our mathematical approach.

The Helmholtz equation (1.1) has been studied already by Euler and Lagrange, but Helmholtz was the first who expressed solutions in bounded domains with a representation formula [18]. In unbounded domains, one faces the problem of boundary conditions at infinity. We recall that two fundamental solutions of the Helmholtz equation for $x \in \mathbb{R}^{3}$ are given by

$$
u_{\text {out }}(x):=\frac{1}{|x|} e^{i \omega|x|} \quad \text { and } \quad u_{\text {inc }}(x):=\frac{1}{|x|} e^{-i \omega|x|} .
$$

With the time-dependence $e^{-i \omega t}$, the solution $u_{\text {out }}$ represents an outgoing wave, $u_{\text {inc }}$ an incoming wave. Outgoing waves are expected to be the building stones of solutions of scattering problems, incoming waves should not be present in the expansion of solutions.

Sommerfeld introduced in [37] for dimension $n=3$ a radiation condition; until today, it is the standard outgoing wave condition in free space and is named after him:

$$
|x|^{(n-1) / 2}\left(\partial_{|x|} u-i \omega u\right)(x) \rightarrow 0 \quad \text { as }|x| \rightarrow \infty .
$$

The solution $u_{\text {out }}$ satisfies (1.4) and is therefore admissible, $u_{\text {inc }}$ does not satisfy (1.4) and is not accepted as a solution. Sommerfeld justified his radiation condition with a uniqueness proof: Prescribing boundary data on an obstacle (the scatterer) and the radiation condition (1.4) at infinity, the Helmholtz equation has at most one solution. Actually, Sommerfeld demanded two further properties to guarantee uniqueness, but the results of Rellich (today known as "Rellich Lemma") showed that the additional assumptions are not necessary [34], see [35] for the historical background.

For two reasons, we cannot use the Sommerfeld radiation condition. The first is that we consider $x$-dependent coefficients $a$. The interest in $x$-dependent coefficients is not new: Sommerfeld himself studied the case that $a$ takes two different values in two disjoint half-spaces, Jäger studied in [21] coefficients $a$ that stabilize to constant coefficients for $|x| \rightarrow \infty$. Our situation is different, since $a$ is periodic in the right 
half plane. The second reason is that we study a waveguide such that, in the above sense, our situation is neither one- nor two-dimensional. For constant coefficients, the elementary solution in a strip $\mathbb{R} \times(0,1)$ is $e^{i \kappa \cdot x}$ with $\kappa=\left(\kappa_{1}, \kappa_{2}\right)$ and $|\kappa|^{2}=\omega$, which is right-going for $\kappa_{1}>0$ and left-going for $\kappa_{1}<0$. The solution has no decay (similar to the one-dimensional case), but the expression $\partial_{x_{1}} u-i \omega u$ does not vanish for right-going waves due to the presence of $\kappa_{2}$.

The idea of our outgoing wave condition is simple: Every function on a rectangular domain can be expanded in Bloch waves. We demand that the expansion of the solution contains only outgoing waves. The precise form of the outgoing wave condition is slightly more technical since we have to consider restrictions of the solution $u$ to large rectangles (in order to have a small contribution of non-periodicity effects).

We emphasize that, even though the conditions become more technical, we follow the historical pathway: The expression in (1.4) can be understood as a projection of the solution $u$ onto incoming waves. Outgoing waves are filtered out and it is demanded that the remainder is small for large radii. Our outgoing wave condition (3.6) is: At the far right, the solution $u$ can be expanded in a Bloch series that contains only rightgoing waves. With this requirement, we follow once more Sommerfeld who writes in [37]: "at infinity $u$ must be representable as a sum (or integral) of waves of the divergent traveling type." For an extensive study of homogeneous media we refer to [11].

\section{On radiation in waveguides and photonic crystals}

An important contribution is the recent work of Fliss and Joly [16], which is also concerned with outgoing wave conditions and the existence and the uniqueness of solutions for periodic wave-guides. Essentially, the outgoing wave condition of [16] (for $x_{1} \rightarrow+\infty$ ) reads

$$
u(x)=\sum_{\lambda \in N(\omega)} \alpha_{\lambda}^{+} U_{\lambda}^{+}(x)+w^{+}(x),
$$

where $N(\omega)$ is a finite index set, $\alpha_{\lambda}^{+}$are real coefficients, $U_{\lambda}^{+}$are right-going Bloch waves and $w^{+}(x)$ is exponentially small for $x_{1} \rightarrow+\infty$. The setting of the problem differs in one important point from ours: [16] studies a medium which is identical at the far left and at the far right, which allows to use global Floquet-Bloch transformations; this is not possible in our setting. Below we give a more detailed comparison of our results to those of [16].

Another radiation condition in a waveguide with varying index in transversal direction is studied in [6]. The "modal radiation condition", formulated in Definition 2.4 of [6], demands for solutions $u$ of the Helmholtz equation that

$$
(\mathcal{F} u(x, .))(\lambda)=\hat{\alpha}_{\lambda}^{ \pm} e^{-\sqrt{\lambda}|x|} \quad \text { for } \pm x>a
$$

holds for every $\lambda$. Here, $\mathcal{F}$ denotes a generalized Fourier transform and $x$ is the longitudinal independent variable. As in our radiation condition, it is demanded that only outgoing waves $\left(e^{-\sqrt{\lambda}|x|}\right.$ instead of $\left.e^{+\sqrt{\lambda}|x|}\right)$ are present. The new feature of our approach is that it covers media with oscillations also in longitudinal direction. We cannot use methods that rely on separation of variables and the Fourier transform must be replaced by a Bloch transform. 
Also in [4, the radiation of waves inside a photonic crystal is investigated, and the setting also uses an interface between a photonic crystal and free space. The fundamental difference to our work is that in [4] the underlying frequency $\omega$ is assumed to lie in a band-gap of the photonic crystal. For this reason, waves in [4] are found to decay exponentially in the photonic crystal and no explicit radiation condition must be formulated. We mention [30] and the references therein for other approaches to radiation conditions, also based on Poynting vectors and incoming and outgoing waves.

For numerical calculations, one is interested in replacing the unbounded domain by a bounded domain. In this case, one asks for appropriate boundary conditions that must be imposed on the boundary of the bounded domain. This point of view leads to the construction of Dirichlet-to-Neumann maps or similar ideas [14, 15, 17]. Other key-words are perfectly matched layers [23] or transparent boundary conditions. These approaches may be an alternative to the outgoing wave condition that we suggest here. However, we are not aware of any result for such boundary conditions that implies our uniqueness theorem.

\subsection{Uniqueness and negative refraction}

Following Sommerfeld's example, we accompany our outgoing wave condition in photonic crystals with a uniqueness statement. With this result, we can treat the application on negative refraction. Several problems must be tackled in this process and the uniqueness result is, unfortunately, not as strong and simple as one would like it to be.

It is an essential feature of the Helmholtz equation that, even without source terms and with homogeneous boundary conditions, solutions may be nontrivial. One example is the bounded domain $\Omega=(0,1) \subset \mathbb{R}^{1}$ with the solution $u(x)=\sin (\pi x)$ for $\omega=\pi$. A more relevant example in higher dimension (2 or 3 ) is the Helmholtz resonator: When $\omega$ coincides with the resonance frequency, there is a nontrivial solution to homogeneous boundary conditions, see [36]. For regular exterior domains, the Sommerfeld condition implies uniqueness: The Helmholtz operator has only a continuous spectrum and no point spectrum. We emphasize that this is true only for the Helmholtz equation with constant coefficients.

In our case of non-constant and (looking globally) non-periodic coefficients, there can be nontrivial solutions to the homogeneous Helmholtz equation (satisfying also a radiation condition). In general, such solutions can be localized modes or waves that travel vertically. An example for the first (corresponding to a point spectrum of the operator) are standing waves in a photonic crystals with a point defect, compare e.g. [22], Chapter 5. In the case of a line defect (or in our situation of an interface between free space and photonic crystal), one expects nontrivial solutions travelling along the interface, see e.g. [28, 29]. Concerning the mathematical analysis of defects in a photonic crystal and the possibility that they support modes (and hence act as a waveguide) see [5, 13]. In a vertically periodic setting it was shown in [20] that a line defect cannot support bounded modes.

The strong uniqueness result of [16] is fitting in this background: In a situation where the surrounding medium is perfectly periodic, the radiation condition of [16] implies uniqueness for non-singular frequencies. Vertical waves in the crystal are excluded 
by the non-singularity assumption, waves along a line defect and localized waves are excluded by the absence of defects.

Instead, our uniqueness result for the transmission problem must deal with the fact that the interface can support nontrivial solutions. Furthermore, we want to admit also singular frequencies (allowing for vertical waves in the crystal). Our uniqueness result states: Imposing the new radiation condition in photonic crystals, for non-singular frequencies, every homogeneous solution has a vanishing Bloch measure. Loosely speaking: the solution vanishes far away from the interface. For general frequencies, the radiating solution may contain vertical waves. See Theorem 1.3 for both results.

A more technical problem will accompany us along the way to a radiation condition and to the uniqueness result: The geometry is not globally periodic and the solution $u$ is not periodic (and $u$ is, in general, not periodic on any rectangle in the right half plane). For this reason, neither a Bloch transformation of $u$ nor a periodic Bloch expansion of $u$ are meaningful as such. We will have to truncate $u$ on large squares at the far right and consider the Bloch expansion of the result. We must use large squares in order to achieve that the truncation process introduces only small errors.

Bloch measures (as used e.g. in [1], pp. 182-183) are the appropriate tool for the limit analysis, which is necessary for the following reason: A periodic Bloch expansion uses a discrete set of frequences $j$. In general, not even the elementary frequency condition $\mu_{0}(j)=\omega^{2}$ (the Bloch wave frequency coincides with the frequency of the Helmholtz equation) can be satisfied in a discrete set of frequencies $j$. For this reason, we cannot expect that the Bloch expansion of $u$ (at a finite distance) satisfies conditions such as $\mu_{0}(j)=\omega^{2}$. Instead, we must introduce a limiting object (the Bloch measure). Our aim is to derive properties of this limiting object.

Regarding other mathematical approaches to related problems, we mention [2, 3], where the authors investigate diffraction effects in time-dependent equations. In [1], the spectrum of an elliptic operator in a periodic medium is investigated. We use some methods of [1], in particular in the pre-Bloch expansion. Moreover, the above mentioned problem of waves that are concentrated at the interface of the photonic crystal has a counterpart in [1]: The part of the spectrum that is related to the boundary layer cannot be characterized explicitly (in the sense of [1], where the sequence $\varepsilon_{i} \rightarrow 0$ is fixed, and in contrast to [9], where the sequence $\varepsilon_{i} \rightarrow 0$ is chosen appropriately).

We close this section with more references to negative refraction effects. Negative refraction can be a consequence of a negative index material, see [31] for the effect and [7, 8, 10, 25, 26] for rigorous results, obtained with the tools of homogenization theory. In [12, 32, the negative refraction effect is explained in the spirit of negative index materials. But negative refraction can also occur without a negative index material, see [27]. We note that the photonic crystals in [12] and in [27] are identical and that they do not have a negative effective index in the sense of homogenization. With the work at hand we support the line of argument of [27]. 


\subsection{Main results}

Throughout this article we consider the following parameters as fixed: The frequency $\omega>0$, the height $h>0$ of the waveguide, the periodicity length $\varepsilon>0$ with $K=$ $h / \varepsilon \in \mathbb{N}$, and the wave number $k \in \mathbb{R}^{2}$ of the incident wave with $k_{2} h \in \varepsilon \mathbb{Z}, k_{1}>0$ and $4 \pi^{2}|k|^{2} / \varepsilon^{2}=\omega^{2}$. The underlying domain is $\Omega:=\mathbb{R} \times(0, h)$ and the coefficient field is $a=a^{\varepsilon}: \Omega \rightarrow \mathbb{R}$. We assume $0<a_{*} \leq a(x) \leq a^{*}<\infty \forall x \in \Omega$ and $a \equiv 1$ on $\left\{x_{1}<0\right\}$, but the latter assumption is not essential. We demand $a \in C^{1}$ with $\varepsilon$-periodicity with respect to $x_{1}$ and $x_{2}$ on $\left\{x_{1}>0\right\}$.

We use Bloch expansions of the solution. Let us give a description of our results, where the superscript " \pm " indicates that we study $\pm x_{1}>0$. The Bloch expansion uses two indices, $m \in \mathbb{N}_{0}=\{0,1,2, \ldots\}$ numbers the eigenfunctions in the periodicity cell and the Bloch number $j \in Z:=[0,1]^{2}$ measures the phase shift along one periodicity cell. We collect the two indices in one index as $\lambda:=(j, m) \in I:=Z \times \mathbb{N}_{0}$. To every $\lambda \in I$ we associate a Poynting number $P_{\lambda}^{ \pm} \in \mathbb{R}$, see $(3.1)$. For the Bloch wave $U_{\lambda}^{ \pm}$with index $\lambda$, the number $P_{\lambda}^{ \pm}$is a measure for the flux of energy in positive $x_{1}$-direction.

We introduce the outgoing wave condition (on the right)

$$
f_{R Y_{\varepsilon}}\left|\Pi_{<0}^{+}\left(u_{R}^{+}\right)\right|^{2} \rightarrow 0 \quad \text { as } \quad R \rightarrow \infty
$$

Here $u_{R}^{+}$is, up to periodic extensions, the function $u_{R}^{+}\left(x_{1}, x_{2}\right)=u\left(R \varepsilon+x_{1}, x_{2}\right)$. The map $\Pi_{<0}^{+}$is a projection onto those Bloch waves that correspond to an energy flux to the left (i.e. incoming waves, $P_{\lambda}^{+}<0$ ). The precise description is given in Definition 3.3. The outgoing condition on the left is analogous; Bloch waves that correspond to an energy flux to the right (i.e. incoming waves, $P_{\lambda}^{-}>0$ ) are excluded on the far left.

Our results are formulated with the help of index sets. Waves with vertical energy flux (or no energy flux) correspond to $\lambda \in I:=Z \times \mathbb{N}_{0}$ in

$$
I_{=0}^{ \pm}:=\left\{\lambda \in I \mid P_{\lambda}^{ \pm}=0\right\},
$$

and, for a given $m \in \mathbb{N}_{0}$, to $j \in Z=[0,1]^{2}$ in the index set

$$
J_{=0, m}^{ \pm}:=\left\{j \in Z \mid P_{(j, m)}^{ \pm}=0\right\}=\left\{j \in Z \mid(j, m) \in I_{=0}^{ \pm}\right\}
$$

The statements below are meaningful for general frequencies $\omega>0$. Unfortunately, we are only able to prove theorems for moderate frequencies, as expressed in the following assumption. It demands that the frequency of the wave is below the energy band corresponding to the index $m=1$ (below the second band).

Assumption 1.1 (Smallness of the frequency). We assume on the coefficient a and the frequency $\omega$ that

$$
\omega^{2}<\inf _{j \in Z, m \geq 1} \mu_{m}^{+}(j),
$$

and $\omega^{2}<\inf _{j \in Z, m \geq 1} \mu_{m}^{-}(j)$, where $\mu_{m}^{ \pm}(j)$ are the Bloch-eigenvalues.

Our results concern solutions $u$ of the transmission problem, specified as follows. 
Problem 1.2 (Transmission problem). We say that $u \in H_{\mathrm{loc}}^{1}(\Omega)$ solves the scattering problem if it satisfies the Helmholtz equation (1.1) in $\Omega=\mathbb{R} \times(0, h)$ with $h=K \varepsilon$ and periodic boundary conditions in the $x_{2}$-variable. We furthermore assume that it is generated by the incoming wave $U_{\mathrm{inc}}$ of (1.2) in the following sense: $u$ satisfies the outgoing wave condition (3.6) on the right and the difference $u-U_{\text {inc }}$ satisfies the outgoing wave condition (3.7) on the left.

Our uniqueness result characterizes the Bloch measures $\nu_{l, \infty}^{ \pm}$of a difference of two solutions (the Bloch measures are introduced in Definition 4.2). The theorem below yields that, for large values of $\left|x_{1}\right|$, the difference of two solutions does not contain Bloch waves with an eigenvalue index larger than 0. Furthermore, only those waves can appear that satisfy all of the following three requirements: They correspond to the imposed frequency $\omega$, they correspond to vertically periodic waves, they transport energy in vertical direction.

Theorem 1.3 (Uniqueness). Let Assumption 1.1 on the frequency $\omega$ be satisfied. For the incoming wave $U_{\text {inc }}$ with wave vector $k$, let $u$ and $\tilde{u}$ be two solutions of the transmission Problem 1.2. For $l \in \mathbb{N}_{0}$, let $\nu_{l, \infty}^{ \pm}$be the Bloch measures that are generated by the difference $v:=u-\tilde{u}$. Then:

$$
\begin{aligned}
\nu_{l, \infty}^{ \pm} & =0 \quad \text { for } l \geq 1, \\
\operatorname{supp}\left(\nu_{0, \infty}^{ \pm}\right) & \subset\left\{j \in Z \mid \mu_{0}^{ \pm}(j)=\omega^{2}, j_{2} \in \mathbb{Z} / K\right\} \cap J_{=0,0}^{ \pm} .
\end{aligned}
$$

An immediate consequence of Theorem 1.3 is the following result for frequencies $\omega$ that do not support vertical waves.

Corollary 1.4 (Uniqueness for non-singular frequencies). Let the situation be as in Theorem 1.3 and let the frequency $\omega$ be non-singular in the sense that $\left\{j \in Z \mid \mu_{0}^{ \pm}(j)=\omega^{2}, j_{2} \in \mathbb{Z} / K\right\} \cap J_{=0,0}^{ \pm}=\emptyset$. Then the difference $v:=u-\tilde{u}$ of two solutions of the transmission Problem 1.2 has a vanishing Bloch measure.

Our second main result shows that the transmission of an incoming wave occurs in such a way that two quantities are conserved: The vertical wave number and the energy.

Theorem 1.5 (Transmission conditions). Let Assumption 1.1 be satisfied, let $k$ be the wave vector of the incoming wave $U_{\mathrm{inc}}$. Let $u$ be a solution of the transmission problem 1.2 and let $\nu_{l, \infty}^{ \pm}$, with $l \in \mathbb{N}_{0}$, be the Bloch measures that are generated by $u$. Then $\nu_{l, \infty}^{ \pm}=0$ for $l \geq 1$ and

$$
\operatorname{supp}\left(\nu_{0, \infty}^{ \pm}\right) \subset\left\{j \in Z \mid \mu_{0}^{ \pm}(j)=\omega^{2}, j_{2} \in \mathbb{Z} / K\right\} \cap\left(\left\{j \in Z \mid j_{2}=k_{2}\right\} \cup J_{=0,0}^{ \pm}\right) .
$$

As above, we have the following corollary for non-singular frequencies.

Corollary 1.6 (Transmission condition for non-singular frequencies). Let the situation be as in Theorem 1.5 and let the frequency $\omega$ be non-singular. Then the Bloch measure $\nu_{0, \infty}^{ \pm}$of u satisfies

$$
\operatorname{supp}\left(\nu_{0, \infty}^{ \pm}\right) \subset\left\{j \in Z \mid \mu_{0}^{ \pm}(j)=\omega^{2} \text { and } j_{2}=k_{2}\right\} .
$$




\subsection{Further comments on the main results}

On the uniqueness result. We recall that we expect the existence of solutions that are supported on the interface between photonic crystal and free space. For this reason, uniqueness results can only provide information "far away from the interface", i.e. information on the Bloch measure.

A weakness of our uniqueness results concerns Assumption 1.1: Our results are proven under the assumption that the underlying frequency $\omega$ is in the first band (more precisely: below the second band). Our conjecture is that our uniqueness result remains valid for arbitrary frequencies, stating that $\operatorname{supp}\left(\nu_{l, \infty}^{ \pm}\right) \subset\left\{j \mid \mu_{l}^{ \pm}(j)=\omega^{2}, j_{2} \in \mathbb{Z} / K\right\} \cap$ $J_{=0, l}^{ \pm}$for every $l \geq 0$. Due to a lack of orthogonality properties in the sesquilinear form $b$ (see Section 4), we must exploit the frequency assumption in our uniqueness proof.

Relations to Fliss and Joly [16]. The contribution [16] contains strong results: 1. A uniqueness result in the classical form (due to the absence of an interface that can support waves and due to the restriction to non-singular frequencies). 2. An existence result, based on a limiting absorption principle. We note that also the existence result of [16] uses global Floquet-Bloch transformations and is therefore not easily adaptable to our setting. We remark that our outgoing wave condition is weaker than the one of [16], see Lemma 3.8. This means that, apart from the problems due to the non-periodic geometry, an existence proof should be simpler for our outgoing wave condition.

We mention at this place that our outgoing wave condition differs in one point with all existing radiation conditions: Our condition does not use explicitely the frequency $\omega$. We regard this as an advantage: our condition might be applicable also in timedependent problems.

$\boldsymbol{A}$ possible scaling in $\varepsilon>0$. In all our theorems we keep the length scale $\varepsilon>0$ fixed. In other words: the wave-length $1 / \omega$ and the periodicity length $\varepsilon$ are both of order 1. It is very interesting to analyze the behavior of light in small micro-structures, i.e. to analyze the limit $\varepsilon \rightarrow 0$. The limit can be performed in two settings: In the classical homogenization problem, one keeps $\omega$ (and hence the wave-length) fixed and analyzes the behavior of solutions $u=u^{\varepsilon}$ as $\varepsilon \rightarrow 0$. This approach was carried out e.g. in [7, 8, 10, 25, 26].

The second setting regards the limit $\varepsilon \rightarrow 0$ in a situation where the wave-length of the incoming wave is also of order $\varepsilon$. This is the scaling that is suggested by our notation in $(1.2)$, which corresponds to a frequency $\omega=\omega^{\varepsilon}=\varepsilon^{-1} \omega^{*}$. Loosely speaking, our Theorem 1.5 yields in this scaling: Solutions $u^{\varepsilon}$ to the scattering problem with incoming wave (1.2) for fixed $k$ consist, at a fixed distance $x_{1}>0$ from the interface and in the limit $\varepsilon \rightarrow 0$, only of Bloch waves that correspond to the frequency $\omega^{\varepsilon}$ and to the wave number $k_{2}$ (up to vertical waves).

Outline of this contribution. Bloch expansions are described in Section 2, In Section 3 we define energy flux numbers and corresponding index sets; these are used to define the new outgoing wave condition. In Section 4 we define Bloch measures, Theorems 1.3 and 1.5 are shown in Section 4.3 . 


\section{Bloch expansions}

\subsection{Pre-Bloch expansions}

We start our analysis with a discrete expansion. This discrete expansion is the first stage of a Bloch expansion and closely related to the Floquet-Bloch transform. We apply it to the $h$-periodic function $u\left(x_{1}, \cdot\right)$. The subsequent result appears as Lemma 4.9 in [1].

Lemma 2.1 (Vertical pre-Bloch expansion). Let $K \in \mathbb{N}$ be the number of periodicity cells and let $h=\varepsilon K$ be the height of the strip $\mathbb{R} \times(0, h)$. Let $u \in L_{\mathrm{loc}}^{2}(\mathbb{R} \times(0, h) ; \mathbb{C})$ be a function. Then $u$ can be expanded uniquely in periodic functions with phase-shifts: With the finite index set $Q_{K}:=\left\{0, \frac{1}{K}, \frac{2}{K}, \ldots, \frac{K-1}{K}\right\}$ we find

$$
u\left(x_{1}, x_{2}\right)=\sum_{j_{2} \in Q_{K}} \Phi_{j_{2}}\left(x_{1}, x_{2}\right) e^{2 \pi i j_{2} x_{2} / \varepsilon},
$$

where each function $\Phi_{j_{2}}\left(x_{1}, \cdot\right)$ is E-periodic. The equality (2.1) holds in $L_{\text {loc }}^{2}(\mathbb{R} \times$ $(0, h) ; \mathbb{C})$.

Sketch of proof. We sketch a proof (different from the one chosen in [1]), considering only $u=u\left(x_{2}\right)$ and $h=1$. Expanding $u$ in a Fourier series, we may write

$$
u\left(x_{2}\right)=\sum_{k_{2} \in \varepsilon \mathbb{Z}} \beta_{k_{2}} e^{2 \pi i k_{2} x_{2} / \varepsilon} .
$$

For every $j_{2} \in \varepsilon \mathbb{N}_{0}$ with $j_{2}<1$ (i.e. for every $j_{2} \in Q_{K}$ ) we set

$$
\Phi_{j_{2}}\left(x_{2}\right):=\sum_{k_{2} \in j_{2}+\mathbb{Z}} \beta_{k_{2}} e^{2 \pi i\left(k_{2}-j_{2}\right) x_{2} / \varepsilon} .
$$

With this choice, each $\Phi_{j_{2}}$ is $\varepsilon$-periodic and (2.1) is satisfied.

For the above pre-Bloch expansion we define the projection on a vertical wave number $k_{2}$ as follows.

Definition 2.2 (Vertical pre-Bloch projection $\left.\Pi_{k_{2}}^{\mathrm{vert}}\right)$. Let $u \in L_{\text {loc }}^{2}(\mathbb{R} \times(0, h) ; \mathbb{C}$ ) with $h=\varepsilon K$ be a function on a strip and let $k_{2} \in Q_{K}$ be a vertical wave number. Then, expanding $u$ as in (2.1), we set

$$
\Pi_{k_{2}}^{\mathrm{vert}} u\left(x_{1}, x_{2}\right):=\Phi_{k_{2}}\left(x_{1}, x_{2}\right) e^{2 \pi i k_{2} x_{2} / \varepsilon} .
$$

The projection is an orthogonal projection: For $\varepsilon$-periodic functions $\Phi$ and $\tilde{\Phi}$ and indices $k_{2} \neq \tilde{k}_{2}$ there holds $\int_{0}^{h} \overline{\Phi\left(x_{2}\right)} e^{-2 \pi i \tilde{k}_{2} x_{2} / \varepsilon} \tilde{\Phi}\left(x_{2}\right) e^{2 \pi i k_{2} x_{2} / \varepsilon} d x_{2}=0$ by Lemma A.1.

We will later use the following fact: If $u$ is a solution of the scattering problem with

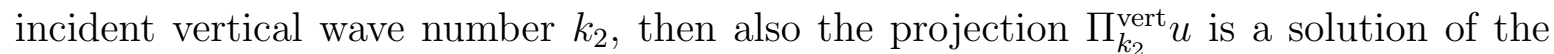
scattering problem. Together with a uniqueness result for solutions, we can conclude from this fact that the vertical wave number is conserved in the photonic crystal.

Below, we have to deal with the following situation: For a function $u$ on a strip with height $h$, we can perform a pre-Bloch expansion. We may also extend $u$ periodically in the vertical direction and perform a pre-Bloch expansion of the extended function on a wider strip. We find that both constructions yield the same result. 
Remark 2.3 (Vertical pre-Bloch expansion of a periodically extended function). Let $K=h / \varepsilon \in \mathbb{N}$ denote the number of periodicity cells in vertical direction and let $u \in$ $L_{\mathrm{loc}}^{2}(\mathbb{R} \times(0, h))$ be a function with vertical pre-Bloch expansion

$$
u\left(x_{1}, x_{2}\right)=\sum_{j_{2} \in Q_{K}} \Phi_{j_{2}}\left(x_{1}, x_{2}\right) e^{2 \pi i j_{2} x_{2} / \varepsilon}
$$

Let $R \in \mathbb{N}$ be a multiple of $K$ and let $\tilde{u}$ be the periodic extension of $u$ to the interval $(0, \varepsilon R)$ in $x_{2}$-direction. Then $\tilde{u} \in L_{\text {loc }}^{2}(\mathbb{R} \times(0, \varepsilon R))$ has the vertical pre-Bloch expansion

$$
\tilde{u}\left(x_{1}, x_{2}\right)=\sum_{\tilde{j}_{2} \in Q_{R}} \tilde{\Phi}_{\tilde{j}_{2}}\left(x_{1}, x_{2}\right) e^{2 \pi i \tilde{j}_{2} x_{2} / \varepsilon},
$$

where the coefficients according to the finer grid $Q_{R}$ satisfy

$$
\tilde{\Phi}_{\tilde{j}_{2}}(x)=\left\{\begin{aligned}
0 & \text { if } \tilde{j}_{2} \notin Q_{K}, \\
\Phi_{\tilde{j}_{2}}(x) & \text { if } \tilde{j}_{2} \in Q_{K} .
\end{aligned}\right.
$$

The statement follows immediately from the uniqueness of the pre-Bloch expansion. Remark 2.3 explains our choice concerning scalings: Given a sequence of functions $u_{R}$, defined on a sequence of increasing domains, at first sight, one might find it natural to rescale $u_{R}$ to a standard domain and to analyze the sequence of rescaled functions. Instead, we work with the sequence $u_{R}$ on increasing domains. In this way, one index $j \in Z$ always refers to the same elementary wave, which allows to investigate the Bloch measure limit.

Pre-Bloch expansion in two variables. For a function $u$ that is defined on a rectangle and that is periodic in both directions, the pre-Bloch expansion in two variables can be defined by expanding first in one variable and then in the other.

For functions $u$ on $\mathbb{R} \times(0, h)$ the situation is more difficult, since $u$ is not periodic in $x_{1}$-direction. In order to expand in both directions, we truncate $u$ with a cut-off function $\eta: \mathbb{R} \times[0, h]$ with compact support. For convenience, we assume that the support of $\eta$ is contained in the square $[0, h] \times[0, h]$.

The truncation of $u$ is defined as $w(x):=u(x) \eta(x)$. We expand $w$ (on the square $[0, h] \times[0, h])$ in both directions in a pre-Bloch expansion, using the vector $j=\left(j_{1}, j_{2}\right) \in$ $Q_{K} \times Q_{K}$ and $x=\left(x_{1}, x_{2}\right)$ :

$$
w(x)=\sum_{j \in Q_{K} \times Q_{K}} \Phi_{j}(x) e^{2 \pi i j \cdot x / \varepsilon} .
$$

The functions $\Phi_{j}=\Phi_{\left(j_{1}, j_{2}\right)}$ are now $\varepsilon$-periodic in both variables. Due to orthogonality there holds $(h=\varepsilon K)$

$$
\frac{1}{(\varepsilon K)^{2}}\|w\|_{L^{2}\left(K Y_{\varepsilon}\right)}^{2}=\sum_{j \in Q_{K} \times Q_{K}} f_{Y_{\varepsilon}}\left|\Phi_{j}\right|^{2},
$$

where $f_{Y_{\varepsilon}}\left|\Phi_{j}\right|^{2}:=\frac{1}{\left|Y_{\varepsilon}\right|} \int_{Y_{\varepsilon}}\left|\Phi_{j}\right|^{2}$ denotes the mean value. 


\subsection{Bloch expansion}

With the help of the pre-Bloch expansion we construct now the Bloch expansion. This step consists in developing each of the periodic functions $\Phi_{j}$ for $j=\left(j_{1}, j_{2}\right)$ in terms of eigenfunctions of the operator

$$
\mathcal{L}_{j}^{+}:=-(\nabla+2 \pi i j / \varepsilon) \cdot\left(a^{\varepsilon}(x)(\nabla+2 \pi i j / \varepsilon)\right) .
$$

The operator $\mathcal{L}_{j}^{+}$acts on complex-valued functions on the cell $Y_{\varepsilon}$ with periodic boundary conditions. It appears in the analysis of 1.1 for the following reason: Let $\Psi_{j}^{+}$be an eigenfunction of $\mathcal{L}_{j}^{+}$with eigenvalue $\mu^{+}(j)$; then there holds

$$
-\nabla \cdot\left(a^{\varepsilon}(x) \nabla\left[\Psi_{j}^{+} e^{2 \pi i j \cdot x / \varepsilon}\right]\right)=\left[\mathcal{L}_{j}^{+} \Psi_{j}^{+}\right] e^{2 \pi i j \cdot x / \varepsilon}=\mu^{+}(j)\left[\Psi_{j} e^{2 \pi i j \cdot x / \varepsilon}\right] .
$$

We see that $\Psi_{j}^{+} e^{2 \pi i j \cdot x / \varepsilon}$ is a solution of the Helmholtz equation on the right half-plane if and only if $\mu^{+}(j)=\omega^{2}$.

We have to distinguish between $x_{1}>0$ and $x_{1}<0$. On the right, the expansion is performed with $\mathcal{L}_{j}^{+}$as above, with the periodic coefficient $a^{\varepsilon}=a^{\varepsilon}(x)$. On the left, expansions are performed according to $a^{\varepsilon} \equiv 1$ with the operator $\mathcal{L}_{j}^{-}:=-(\nabla+2 \pi i j / \varepsilon)$. $(\nabla+2 \pi i j / \varepsilon)$. The result is a classical Fourier expansion of the solution.

Definition 2.4 (Bloch eigenfunctions). Let $j \in[0,1]^{2}$ be a fixed wave vector. We denote by $\left(\Psi_{j, m}^{+}\right)_{m \in \mathbb{N}_{0}}$ the family of eigenfunctions of the operator $\mathcal{L}_{j}^{+}$of (2.7). The labelling is such that the corresponding eigenvalues $\mu_{m}^{+}(j)$ are ordered, $\mu_{m+1}(j) \geq \mu_{m}(j)$ for all $m \in \mathbb{N}_{0}$. Similarly, $\left(\Psi_{j, m}^{-}\right)_{m \in \mathbb{N}_{0}}$ is the family of eigenfunctions of the operator $\mathcal{L}_{j}^{-}$and $\mu_{m}^{-}(j)$ are the corresponding eigenvalues. We normalize with $f_{Y_{\varepsilon}}\left|\Psi_{j, m}^{ \pm}\right|^{2}=1$.

A standard symmetry argument yields that, after an appropriate orthonormalization procedure for multiple eigenvalues, all functions $\Psi_{j, m}^{ \pm}(x) e^{2 \pi i j \cdot x / \varepsilon}$ with $j \in Q_{K}$ are orthonormal in the space $L_{\sharp}^{2}\left(K Y_{\varepsilon} ; \mathbb{C}\right)=L^{2}\left(K Y_{\varepsilon} ; \mathbb{C}\right)$ (the sharp symbol is sometimes used to indicate that one thinks of periodic functions, but, of course, in the case of $L^{2}$, the periodicity does not alter the function space). On the left hand side (i.e. for $x_{1}<0$, denoted with the superscript "-"), the Bloch eigenfunctions are harmonic waves and the Bloch expansion coincides with a Fourier expansion. We collect properties on the left half-domain in Remark 3.6.

Lemma 2.5 (Bloch expansion). Let $K \in \mathbb{N}$ be the number of cells in each direction, let $u \in L^{2}\left(K Y_{\varepsilon} ; \mathbb{C}\right)$ be a function on the square $(0, K \varepsilon) \times(0, K \varepsilon)$. Expanding $u$ in a pre-Bloch expansion and then expanding each $\Phi_{j}$ in eigenfunctions $\Psi_{j, m}^{+}$we obtain, with coefficients $\alpha_{j, m}^{+} \in \mathbb{C}$,

$$
u(x)=\sum_{j \in Q_{K} \times Q_{K}} \sum_{m=0}^{\infty} \alpha_{j, m}^{+} \Psi_{j, m}^{+}(x) e^{2 \pi i j \cdot x / \varepsilon},
$$

and similarly, for an expansion corresponding to constant coefficients $a^{\varepsilon} \equiv 1$,

$$
u(x)=\sum_{j \in Q_{K} \times Q_{K}} \sum_{m=0}^{\infty} \alpha_{j, m}^{-} \Psi_{j, m}^{-}(x) e^{2 \pi i j \cdot x / \varepsilon} .
$$


To shorten notation, we will use the multi-index $\lambda=(j, m)$ in the index-set $I_{K}:=$ $\left\{(j, m) \mid j \in Q_{K} \times Q_{K}, m \in \mathbb{N}_{0}\right\} \subset I:=Z \times \mathbb{N}_{0}$. Abbreviating additionally

$$
U_{\lambda}^{ \pm}(x):=\Psi_{\lambda}^{ \pm}(x) e^{2 \pi i j \cdot x / \varepsilon},
$$

we may write the formulas of Lemma 2.5 as

$$
u(x)=\sum_{\lambda=(j, m) \in I_{K}} \alpha_{\lambda}^{ \pm} \Psi_{\lambda}^{ \pm}(x) e^{2 \pi i j \cdot x / \varepsilon}=\sum_{\lambda \in I_{K}} \alpha_{\lambda}^{ \pm} U_{\lambda}^{ \pm}(x) .
$$

The expansion holds for the basis functions $U_{\lambda}^{+}$with coefficients $\alpha_{\lambda}^{+}$and for the basis functions $U_{\lambda}^{-}$with coefficients $\alpha_{\lambda}^{-}$. Moreover, due to $L^{2}$-orthonormality of the functions $U_{\lambda}^{ \pm}$, with $h=\varepsilon K$ and $K Y_{\varepsilon}=(0, h) \times(0, h)$,

$$
\frac{1}{(\varepsilon K)^{2}}\|u\|_{L^{2}\left(K Y_{\varepsilon}\right)}^{2}=\sum_{\lambda \in I_{K}}\left|\alpha_{\lambda}^{ \pm}\right|^{2} .
$$

\section{Outgoing wave condition}

\subsection{Poynting numbers and projections}

Let $\lambda=(j, m) \in I$ be an index and let $U_{\lambda}^{ \pm}$be the corresponding Bloch function. Denoting by $e_{1}=(1,0) \in \mathbb{R}^{2}$ the first unit vector, we connect to $\lambda \in I$ the real numbers

$$
\begin{aligned}
& P_{\lambda}^{+}:=\operatorname{Im} \int_{Y_{\varepsilon}} \bar{U}_{\lambda}^{+}(x) e_{1} \cdot\left[a^{\varepsilon}(x) \nabla U_{\lambda}^{+}(x)\right] d x, \\
& P_{\lambda}^{-}:=\operatorname{Im} \int_{Y_{\varepsilon}} \bar{U}_{\lambda}^{-}(x) e_{1} \cdot \nabla U_{\lambda}^{-}(x) d x .
\end{aligned}
$$

The number $P_{\lambda}^{+}$is related to the Poynting vector of the Bloch eigenfunction $U_{\lambda}^{+}$. It corresponds (up to a positive constant) to the horizontal group velocity of this eigenfunction and measures its energy flux in horizontal direction: In the case $P_{\lambda}^{+}>0$, the energy of the wave is travelling to the right, in the case $P_{\lambda}^{+}<0$, the energy of the wave is travelling to the left.

Let us point out the relation to Maxwell's equations: If $u$ denotes the out-ofplane magnetic field, i.e. $H=(0,0, u)$, then the electric field is $\left(E_{1}, E_{2}, 0\right)$ with $E_{1}=(-i \omega \epsilon)^{-1} \partial_{2} u$ and $E_{2}=(i \omega \epsilon)^{-1} \partial_{1} u$ where $\epsilon$ is the permittivity of the medium. The complex Poynting vector is $P=\frac{1}{2} E \times \bar{H}$, so the real part of its horizontal component is $\operatorname{Re}\left(e_{1} \cdot P\right)=\operatorname{Re}\left(\frac{1}{2} \bar{H}_{3} E_{2}\right)=(2 \omega)^{-1} \operatorname{Re}\left(-i \bar{u} \epsilon^{-1} \partial_{1} u\right)=(2 \omega)^{-1} \operatorname{Im}\left(\bar{u} a \partial_{1} u\right)$, where we used that the coefficient $a=\epsilon^{-1}$ is the inverse permittivity. Our expression in (3.1) coincides up to the factor $2 \omega$ with an integral of this expression. Since $P$ represents a local energy flux, the physical quantity of a total energy flux is a surface integral over $P$. In fact, for solutions $U_{\lambda}$ of a Helmholtz equation, the surface integral is independent of the position of the surface. Hence our volume integral in 3.1 indeed coincides with the physical quantity of a surface integral.

The index set for $\lambda$ : In our construction, we fix the height $h>0$ of the domain and the periodicity length $\varepsilon=h / K$, the Bloch expansion is performed in this fixed 
geometry. As a consequence, we consider only indices $\lambda=(j, m) \in I_{K}$, the frequency parameter $j$ must lie in the discrete set $Q_{K} \times Q_{K} \subset Z$. On the other hand, for arbitrary $j \in Z$, we can still consider the functions $\Psi_{j, m}^{ \pm}$and $U_{\lambda}^{ \pm}$. They do not depend on $K$, hence also the values $P_{\lambda}^{ \pm}$are independent of $K$.

Definition 3.1 (Index sets and projections). We define the set of indices corresponding to right-going waves in $x_{1}>0$ as

$$
I_{>0}^{+}:=\left\{\lambda \in I \mid P_{\lambda}^{+}>0\right\}
$$

The index sets $I_{>0}^{-}, I_{<0}^{ \pm}, I_{\geq 0}^{ \pm}, I_{\leq 0}^{ \pm}, I_{=0}^{ \pm}$are defined accordingly.

For $K \in \mathbb{N}$ we define the projections $\Pi_{>0}^{ \pm}$as follows: Let $u \in L^{2}\left(K Y_{\varepsilon} ; \mathbb{C}\right)$ be a function with the discrete Bloch expansion

$$
u(x)=\sum_{\lambda \in I_{K}} \alpha_{\lambda}^{ \pm} U_{\lambda}^{ \pm}(x) .
$$

Then we set

$$
\Pi_{>0}^{ \pm} u(x):=\sum_{\lambda \in I_{K} \cap I_{>0}^{ \pm}} \alpha_{\lambda}^{ \pm} U_{\lambda}^{ \pm}(x) .
$$

With this definition, $\Pi_{>0}^{ \pm}$are the projections onto right-going Bloch-waves. The projections $\Pi_{<0}^{ \pm}, \Pi_{\geq 0}^{ \pm}, \Pi_{\leq 0}^{ \pm}$, and $\Pi_{=0}^{ \pm}$are defined accordingly.

For $k_{2} \in Q_{K}$ and $l \in \mathbb{N}_{0}$, the "vertical" projection $\Pi_{k_{2}}^{\mathrm{vert}, \pm}$ and the "eigenvalue" projection $\Pi_{l}^{\mathrm{ev}, \pm}$ are defined by

$$
\begin{aligned}
\Pi_{k_{2}}^{\mathrm{vert}, \pm} u(x) & :=\sum_{\lambda \in\left\{(j, m) \in I_{K} \mid j_{2}=k_{2}\right\}} \alpha_{\lambda}^{ \pm} U_{\lambda}^{ \pm}(x), \\
\Pi_{l}^{\mathrm{ev}, \pm} u(x) & :=\sum_{\lambda \in\left\{(j, m) \in I_{K} \mid m=l\right\}} \alpha_{\lambda}^{ \pm} U_{\lambda}^{ \pm}(x) .
\end{aligned}
$$

Note that the projections $\Pi_{k_{2}}^{\mathrm{vert}, \pm}$ of the discrete Bloch expansion indeed coincide with the projection $\Pi_{k_{2}}^{\mathrm{vert}}$ of the corresponding vertical pre-Bloch expansion of Definition 2.2. The vertical projection is independent of $K$ in the sense that a periodically extended $u$ with a larger value of $K$ has the same projection, compare Remark 2.3 .

\subsection{Bloch expansion at infinity and outgoing wave condition}

We can now formulate the outgoing wave condition for a solution $u$ of the Helmholtz equation (1.1). The loose description of our outgoing wave condition (on the right) is: The Bloch expansion of $u$ does not contain Bloch waves that transport energy to the left.

For a rigorous definition we must deal with the problem that $u$ is not necessarily periodic in $x_{1}$-direction. Our solution to this problem is to consider $u$ on large domains (which reduces the effects of non-periodicity) and to employ a truncation procedure. Furthermore, we want to formulate a condition that characterizes $u$ for large values of 
$x_{1}$. For these two reasons, we consider $u\left(x_{1}, x_{2}\right)$ for $x_{1} \in(R \varepsilon, 2 R \varepsilon)$ with a large natural number $R>>K$.

In order to construct a function on a large domain, we consider the periodic extension of $u$ in vertical direction and restrict afterwards the function to a large rectangle. For convenience of notation, we restrict our analysis to squares.

Definition 3.2 (Bloch expansion far away from the interface). Let $u \in L_{\text {loc }}^{2}(\mathbb{R} \times$ $(0, h) ; \mathbb{C})$ be a function on the infinite strip with height $h=\varepsilon K$. Let $R \in \mathbb{N} K$ be a multiple of $K$. We define $\tilde{u}: \mathbb{R}^{2} \rightarrow \mathbb{C}$ as the h-periodic extension of $u$ in $x_{2}$-direction. We furthermore define functions $u_{R}^{ \pm}: R Y_{\varepsilon} \rightarrow \mathbb{C}$ by

$$
\begin{aligned}
& u_{R}^{+}\left(x_{1}, x_{2}\right):=\tilde{u}\left(R \varepsilon+x_{1}, x_{2}\right), \\
& u_{R}^{-}\left(x_{1}, x_{2}\right):=\tilde{u}\left(-2 R \varepsilon+x_{1}, x_{2}\right) .
\end{aligned}
$$

We use the discrete Bloch expansions of the functions $u_{R}^{ \pm} \in L_{\sharp}^{2}\left(R Y_{\varepsilon} ; \mathbb{C}\right)$,

$$
u_{R}^{ \pm}(x)=\sum_{\lambda \in I_{R}} \alpha_{\lambda, R}^{ \pm} U_{\lambda}^{ \pm}(x)
$$

The coefficients $\left(\alpha_{\lambda, R}^{ \pm}\right)_{\lambda \in I}$ encode the behavior of $u$ for large values of $\left|x_{1}\right|$.

We are now in the position to define the outgoing wave condition for a solution $u$ to the Helmholtz equation, using the short notation $f_{R Y_{\varepsilon}} f:=\frac{1}{\left|R Y_{\varepsilon}\right|} \int_{R Y_{\varepsilon}} f$ for averages of functions.

Definition 3.3 (Outgoing wave condition). For $K \in \mathbb{N}, h=K \varepsilon$, and $R \in \mathbb{N} K$, we consider $u \in L_{\text {loc }}^{2}(\mathbb{R} \times(0, h) ; \mathbb{C})$. We say that u satisfies the outgoing wave condition on the right if the following two conditions are satisfied: $\int_{0}^{h} \int_{L}^{L+1}|u|^{2}$ is bounded, independently of $L \geq 0$, and

$$
f_{R Y_{\varepsilon}}\left|\Pi_{<0}^{+}\left(u_{R}^{+}\right)\right|^{2} \rightarrow 0 \text { as } R \rightarrow \infty .
$$

Accordingly, we say that $u$ satisfies the outgoing wave condition on the left, if $\int_{0}^{h} \int_{L-1}^{L}|u|^{2}$ is bounded, independently of $L \leq 0$, and if

$$
f_{R Y_{\varepsilon}} \mid\left(\left.\Pi_{>0}^{-}\left(u_{R}^{-}\right)\right|^{2} \rightarrow 0 \text { as } R \rightarrow \infty .\right.
$$

Let us repeat the idea of condition (3.6): The function $u$ is considered at the far right by construcing $u_{R}^{+}$as in Definition 3.2 . This function is projected onto the space of left-going waves. We demand that the $L^{2}$-averages of the resulting functions $\Pi_{<0}^{+}\left(u_{R}^{+}\right)$ vanish in the limit $R \rightarrow \infty$.

With the expansion (3.5) we can write condition (3.6) equivalently as:

$$
\sum_{\lambda \in I_{R} \cap I_{<0}^{+}}\left|\alpha_{\lambda, R}^{+}\right|^{2} \rightarrow 0 \text { as } R \rightarrow \infty .
$$

Our aim is to show that this definition of an outgoing wave condition implies uniqueness properties for the scattering problem.

We note that the uniform $L^{2}$-bounds for large values of $|L|$ imply, for solutions $u$ of the Helmholtz equation, also uniform bounds for gradients, see Lemma A.3 in the appendix. 


\subsection{Truncations and $m \geq 1$-projections}

In the outgoing wave condition, we study the limit $\left|x_{1}\right| \sim R \rightarrow \infty$ and the functions $u_{R}^{ \pm}$ on large squares $W_{R}:=R Y_{\varepsilon}=(0, R \varepsilon)^{2}$ with $\left|W_{R}\right|=(\varepsilon R)^{2}$. As a measure for typical values of a function $v$ we use $L^{2}$-averages on $W_{R}$ and the corresponding scalar product,

$$
\langle v, w\rangle_{R}:=f_{W_{R}} v \cdot \bar{w}
$$

In the following we denote by $\mathcal{L}_{0}=\mathcal{L}_{0}^{+}=-\nabla \cdot\left(a^{\varepsilon} \nabla\right)$ the elliptic operator of (2.7). As above, we denote cubes by $W_{R}=R Y_{\varepsilon}$ and, by slight abuse of notation, we write $W_{R-1}:=\varepsilon(1, R-1)^{2}$ for a smaller cube that has the point $\varepsilon(1,1)$ as its bottom left corner and $\varepsilon(R-1, R-1)$ as its top right corner. We use a family of smooth cut-off functions $\eta:=\eta_{R}$ with the properties

$$
\eta_{R} \in C^{\infty}\left(W_{R} ; \mathbb{R}\right), \quad \eta_{R}=1 \text { on } W_{R-1}, \quad\left\|\nabla \eta_{R}\right\|_{\infty} \leq C_{0}, \quad\left\|\nabla^{2} \eta_{R}\right\|_{\infty} \leq C_{0}
$$

for some $R$-independent constant $C_{0}$ ( $\varepsilon>0$ is fixed), and with compact support in $(0, R \varepsilon) \times(0, R \varepsilon)_{\sharp}$, where $(0, R \varepsilon)_{\sharp}$ indicates the interval with identified end points. The latter requirement admits sequences $\eta$ with compact support in $(0, R \varepsilon) \times(0, R \varepsilon)$, but also sequences of vertically periodic functions $\eta$, in particular functions $\eta=\eta\left(x_{1}\right)$. In the subsequent proofs we do not indicate the $R$-dependence of $\eta_{R}$ and write only $\eta$. We furthermore omit the superscipts \pm , the eigenvalue corresponding to $\lambda=(j, m)$ is denoted by $\mu_{\lambda}=\mu_{m}(j)$. Constants are allowed to depend on $\varepsilon>0$.

Lemma 3.4 (The effect of truncations). For $R \in \mathbb{N}$ let $\eta=\eta_{R}$ be a family of cut-off functions satisfying (3.10). Let $v_{R}$ and $w_{R}$ be sequences of functions in $L^{2}\left(W_{R} ; \mathbb{C}\right)$ with $v_{R} \in H^{2}\left(W_{R} ; \mathbb{C}\right)$. We assume that certain averages over boundary strips are bounded:

$$
\frac{1}{R} \int_{W_{R} \backslash W_{R-1}}\left|v_{R}\right|^{2}+\left|\nabla v_{R}\right|^{2} \leq C_{0}, \quad \frac{1}{R} \int_{W_{R} \backslash W_{R-1}}\left|w_{R}\right|^{2} \leq C_{0}
$$

with $C_{0}$ independent of $R$. Then, with a constant $C$ that is independent of $R$ :

1. Application of $\mathcal{L}_{0}$ to a truncated function:

$$
f_{W_{R}}\left|\mathcal{L}_{0}\left(v_{R}\right) \eta-\mathcal{L}_{0}\left(v_{R} \eta\right)\right|^{2}=f_{W_{R}}\left|\mathcal{L}_{0}\left(v_{R}\right) \eta-\sum_{\lambda \in I_{R}} \mu_{\lambda}\left\langle v_{R} \eta, U_{\lambda}\right\rangle_{R} U_{\lambda}\right|^{2} \leq \frac{C}{R} .
$$

2. If $\Pi$ is one of the projections of Definition 3.1, then

$$
f_{W_{R}}\left|\Pi\left(w_{R}\right)-\Pi\left(w_{R} \eta\right)\right|^{2} \leq f_{W_{R}}\left|w_{R}-w_{R} \eta\right|^{2} \leq \frac{C}{R} .
$$

Proof. In the following, the letter $C$ denotes different constants, possibly varying from one line to the next, but always independent of $R$. To prove (3.12), we expand the 
$L^{2}$-function $\mathcal{L}_{0}\left(v_{R}\right) \eta$ in Bloch-waves. The following calculation uses several times integration by parts; due to the $\eta$-factor, no boundary integrals occur. In the first equation we use that the coefficient $\alpha_{\lambda}$ in the expansion of $\mathcal{L}_{0}\left(v_{R}\right) \eta$ is obtained by taking the scalar product with $U_{\lambda}$ (orthonormality of the $U_{\lambda}$ ).

$$
\begin{aligned}
\mathcal{L}_{0}\left(v_{R}\right) \eta & =\sum_{\lambda \in I_{R}}\left\langle\mathcal{L}_{0}\left(v_{R}\right) \eta, U_{\lambda}\right\rangle_{R} U_{\lambda}=\sum_{\lambda \in I_{R}}\left\langle v_{R}, \mathcal{L}_{0}\left(\eta U_{\lambda}\right)\right\rangle_{R} U_{\lambda} \\
& =\sum_{\lambda \in I_{R}}\left(\left\langle v_{R} \eta, \mathcal{L}_{0} U_{\lambda}\right\rangle_{R}+\left\langle v_{R} \mathcal{L}_{0}(\eta), U_{\lambda}\right\rangle_{R}-2\left\langle v_{R} a^{\varepsilon} \nabla \eta, \nabla U_{\lambda}\right\rangle_{R}\right) U_{\lambda} \\
& =\sum_{\lambda \in I_{R}}\left(\mu_{\lambda}\left\langle v_{R} \eta, U_{\lambda}\right\rangle_{R}-\left\langle v_{R} \mathcal{L}_{0}(\eta), U_{\lambda}\right\rangle_{R}+2\left\langle\nabla v_{R} \cdot a^{\varepsilon} \nabla \eta, U_{\lambda}\right\rangle_{R}\right) U_{\lambda} \\
& =\left(\sum_{\lambda \in I_{R}} \mu_{\lambda}\left\langle v_{R} \eta, U_{\lambda}\right\rangle_{R} U_{\lambda}\right)-v_{R} \mathcal{L}_{0}(\eta)+2 a^{\varepsilon} \nabla v_{R} \cdot \nabla \eta,
\end{aligned}
$$

where in the third equality we exploited $\mathcal{L}_{0} U_{\lambda}=\mu_{\lambda} U_{\lambda}$ and $\mu_{\lambda} \in \mathbb{R}$. The contribution of the last two terms can be estimated by

$$
\begin{aligned}
& f_{W_{R}}\left|v_{R} \mathcal{L}_{0}(\eta)\right|^{2}+\left|2 a^{\varepsilon} \nabla v_{R} \cdot \nabla \eta\right|^{2} \\
& \quad \leq\left\|\mathcal{L}_{0}(\eta)\right\|_{L^{\infty}\left(W_{R}\right)}^{2} f_{W_{R}}\left|v_{R}\right|^{2} 1_{\{\operatorname{supp}(\nabla \eta)\}}+\left\|2 a^{\varepsilon} \nabla \eta\right\|_{L^{\infty}\left(W_{R}\right)}^{2} f_{W_{R}}\left|\nabla v_{R}\right|^{2} 1_{\{\operatorname{supp}(\nabla \eta)\}} \\
& \quad \leq \frac{C}{R^{2}}\left(\int_{W_{R} \backslash W_{R-1}}\left|v_{R}\right|^{2}+\int_{W_{R} \backslash W_{R-1}}\left|\nabla v_{R}\right|^{2}\right) \leq \frac{C}{R}
\end{aligned}
$$

In the second inequality we exploited $\operatorname{supp}(\nabla \eta) \subset\left(W_{R} \backslash W_{R-1}\right)$, in the last inequality we used the uniform bounds (3.11). This proves the inquality in (3.12).

Regarding the equality in $(3.12)$ we have to verify that the formal equality $\mathcal{L}_{0} w=$ $\sum_{\lambda} \mu_{\lambda}\left\langle w, U_{\lambda}\right\rangle_{R} U_{\lambda}$ holds for functions $w \in H^{2}\left(W_{R}\right)$ with vanishing boundary data. We find this from

$$
\left\langle\mathcal{L}_{0} w, U_{\lambda}\right\rangle \stackrel{(!)}{=}\left\langle w, \mathcal{L}_{0} U_{\lambda}\right\rangle=\mu_{\lambda}\left\langle w, U_{\lambda}\right\rangle
$$

where we used in the marked equality that boundary terms vanish.

Inequality 3.13 is a direct consequence of linearity and norm-boundedness of the projections:

$$
\begin{aligned}
& f_{W_{R}}\left|\Pi w_{R}-\Pi\left(w_{R} \eta\right)\right|^{2}=f_{W_{R}}\left|\Pi\left(w_{R}(1-\eta)\right)\right|^{2} \\
& \quad \leq f_{W_{R}}\left|w_{R}(1-\eta)\right|^{2} \leq \frac{C}{R^{2}} \int_{W_{R} \backslash W_{R-1}}\left|w_{R}\right|^{2} \leq \frac{C}{R}
\end{aligned}
$$

This concludes the proof.

A warning concerning the non-periodicity of truncated solutions. Let $u$ be a vertically periodic solution of the Helmholtz equation on $\mathbb{R} \times(0, h)$ and let $u_{R}^{+}$be 
defined as in Definition 3.2. Then $u_{R}^{+}$is a solution of the Helmholtz equation on the open square $W_{R}=R Y_{\varepsilon}$. But $u_{R}^{+}$is not a periodic solution on the square (since it is not periodic in horizontal direction).

This fact implies that certain formal calculations are wrong: Let $u_{R}^{+}$have the Bloch expansion $u_{R}^{+}=\sum_{\lambda \in I_{R}} \alpha_{\lambda} U_{\lambda}^{+}$for some coefficients $\alpha_{\lambda}$ (every $L^{2}$-function posesses such an expansion). Then, in general, the following identity fails to hold:

$$
\mathcal{L}_{0} \sum_{\lambda \in I_{R}} \alpha_{\lambda} U_{\lambda}^{+} \stackrel{(?)}{=} \sum_{\lambda \in I_{R}} \alpha_{\lambda} \mathcal{L}_{0}\left(U_{\lambda}^{+}\right)
$$

To see this, let us assume that the relation (?) holds. Then the Helmholtz equation provides $\sum_{\lambda \in I_{R}} \omega^{2} \alpha_{\lambda} U_{\lambda}^{+}=\omega^{2} u_{R}^{+}=\mathcal{L}_{0} u_{R}^{+}=\mathcal{L}_{0} \sum_{\lambda \in I_{R}} \alpha_{\lambda} U_{\lambda}^{+} \stackrel{(?)}{=} \sum_{\lambda \in I_{R}} \alpha_{\lambda} \mathcal{L}_{0}\left(U_{\lambda}^{+}\right)=$ $\sum_{\lambda \in I_{R}} \alpha_{\lambda} \mu_{\lambda}^{+} U_{\lambda}^{+}$. Uniqueness of the Bloch expansion implies $\alpha_{\lambda}\left(\omega^{2}-\mu_{\lambda}^{+}\right)=0$ for every $\lambda \in I_{R}$, hence $\alpha_{\lambda}=0$ for every $\lambda$ with $\mu_{\lambda}^{+} \neq \omega^{2}$. We conclude that the Bloch expansion of $u_{R}^{+}$contains only contributions from those basis functions $U_{\lambda}^{+}$with $\mu_{\lambda}^{+}=\omega^{2}$.

This is a contradiction for general solutions $u$ : Let $\omega$ be a frequency such that $\omega^{2}$ is not contained in the discrete set of $\left(\mu_{\lambda}^{+}\right)_{\lambda \in I_{R}}$. Let furthermore $u$ be a non-vanishing Bloch wave for the frequency $\omega$. Then the expansion of $u$ does not use only $\alpha_{\lambda}=0$, a contradiction.

Lemma 3.5 (Contributions from energy levels $m \geq 1$ ). Let $\omega$ satisfy the smallness condition (1.7) of Assumption 1.1. Let $u \in L_{\text {loc }}^{2}(\mathbb{R} \times(0, h) ; \mathbb{C})$ be a vertically periodic solution of the Helmholtz equation $\mathcal{L}_{0} u=\omega^{2} u$ satisfying the uniform $L^{2}$-bounds of Definition 3.3. Let $\eta=\eta_{R}$ be a family of cut-off functions as in (3.10). Then, with a constant $C$ that is independent of $R$ :

$$
f_{W_{R}}\left|\Pi_{m \geq 1}^{\mathrm{ev}, \pm}\left(u_{R}^{ \pm}\right)\right|^{2} \leq \frac{C}{R} \quad \text { and } \quad f_{W_{R}}\left|\Pi_{m \geq 1}^{\mathrm{ev}, \pm}\left(u_{R}^{ \pm} \eta\right)\right|^{2} \leq \frac{C}{R} .
$$

Proof. We perform the proof for the superscript "+". Relation (3.13) applied to $u_{R}^{+}$ provides

$$
f_{W_{R}}\left|\Pi_{m \geq 1}^{\mathrm{ev},+}\left(u_{R}^{+}\right)-\Pi_{m \geq 1}^{\mathrm{ev},+}\left(u_{R}^{+} \eta\right)\right|^{2} \leq \frac{C}{R} .
$$

Indeed, by the uniform $L^{2}$-bounds of $u$, the condition $\frac{1}{R} \int_{W_{R} \backslash W_{R-1}}\left|u_{R}^{+}\right|^{2} \leq C_{0}$ with $C_{0}$ independent of $R$ is satisfied. The above inequality implies that it is sufficient to show only one of the two relations in (3.15), we show the second.

We now exploit Assumption 1.1. Due to (1.7), there exists $\delta>0$ such that $\mid \omega^{2}-$ $\left.\mu_{\lambda}\right|^{2} \geq \delta$ for all $\lambda=(j, m)$ with $m \geq 1$. We therefore find

$$
\begin{aligned}
& \delta f_{W_{R}}\left|\Pi_{m \geq 1}^{\mathrm{ev},+}\left(u_{R}^{+} \eta\right)\right|^{2}=\delta \sum_{\substack{\lambda=(j, m) \in I_{R} \\
m \geq 1}}\left|\left\langle u_{R}^{+} \eta, U_{\lambda}\right\rangle_{R}\right|^{2} \leq \sum_{\substack{\lambda=(j, m) \in I_{R} \\
m \geq 1}}\left|\left(\omega^{2}-\mu_{\lambda}\right)\left\langle u_{R}^{+} \eta, U_{\lambda}\right\rangle_{R}\right|^{2} \\
& \leq \sum_{\lambda \in I_{R}}\left|\left\langle\omega^{2} u_{R}^{+} \eta, U_{\lambda}\right\rangle_{R}-\left\langle\mu_{\lambda} u_{R}^{+} \eta, U_{\lambda}\right\rangle_{R}\right|^{2}=\sum_{\lambda \in I_{R}}\left|\left\langle\mathcal{L}_{0}\left(u_{R}^{+}\right) \eta, U_{\lambda}\right\rangle_{R}-\left\langle\mu_{\lambda} u_{R}^{+} \eta, U_{\lambda}\right\rangle_{R}\right|^{2}
\end{aligned}
$$




$$
=f_{W_{R}}\left|\mathcal{L}_{0}\left(u_{R}^{+}\right) \eta-\sum_{\lambda \in I_{R}} \mu_{\lambda}\left\langle u_{R}^{+} \eta, U_{\lambda}\right\rangle_{R} U_{\lambda}\right|^{2} \leq \frac{C}{R} .
$$

In the second line we used that $\mathcal{L}_{0}\left(u_{R}^{+}\right)=\omega^{2} u_{R}^{+}$holds pointwise almost everywhere in $W_{R}$. In the last inequality we used (3.12), exploiting the uniform $H^{1}$-bounds provided by Lemma A.3. This concludes the proof.

\subsection{Other radiation conditions and the sesquilinear form $b_{R}^{ \pm}$}

In this section we discuss how our radiation condition simplifies in the case of a homogeneous medium. We furthermore show that the radiation condition suggested by Fliss and Joly in [16] is formally stronger than our condition: Every solution that satisfies the condition of [16] satisfies also our condition. Finally, we introduce the sesquilinear form $b_{R}^{ \pm}$, which plays a major role in our proofs. The form $b_{R}^{ \pm}$can also be used to introduce an even weaker form of the outgoing wave condition.

\section{In free space, Bloch expansions are Fourier expansions}

Let us study the outgoing wave condition in a homogeneous medium. This is our situation for $x_{1}<0$, indicated with the superscript "-". In a homogeneous medium, the Bloch waves are harmonic waves. This allows to give explicit formulas for some quantities and, in particular, for the outgoing wave condition.

Remark 3.6 (Basis functions and Poynting numbers in a homogeneous medium). The functions $\Psi_{j, m}^{-}$are $\varepsilon$-periodic eigenfunctions of the operator $\mathcal{L}_{j}^{-}=-(\nabla+2 \pi i j / \varepsilon)$. $(\nabla+2 \pi i j / \varepsilon)$. As such, for fixed $j \in Z$, they are harmonic waves, $\left\{\Psi_{j, m}^{-} \mid m \in \mathbb{N}_{0}\right\}=$ $\left\{e^{2 \pi i k \cdot x / \varepsilon} \mid k \in \mathbb{Z}^{2}\right\}$. More precisely, for every $j \in Z$ and every $m \in \mathbb{N}$, there exists a wave-number $k=k(j, m) \in \mathbb{Z}^{2}$ such that

$$
\Psi_{j, m}^{-}(x)=e^{2 \pi i k \cdot x / \varepsilon}, \quad \mu_{m}^{-}(j)=4 \pi^{2} \frac{|k+j|^{2}}{\varepsilon^{2}} .
$$

Accordingly, for $\lambda=(j, m)$, we have $U_{\lambda}^{-}(x)=e^{2 \pi i(k+j) \cdot x / \varepsilon}$, and the Poynting number is

$$
P_{\lambda}^{-}=\operatorname{Im} f_{Y_{\varepsilon}} \bar{U}_{\lambda}^{-}(x) e_{1} \cdot \nabla U_{\lambda}^{-}(x) d x=\frac{2 \pi}{\varepsilon}\left(k_{1}+j_{1}\right)
$$

In particular, for the first energy level, $\lambda=(j, 0)$, we find

$$
k=k(j, 0) \in \underset{k \in \mathbb{Z}^{2}}{\operatorname{argmin}}|k+j|^{2},
$$

and thus, for $j=\left(j_{1}, j_{2}\right)$ with $j_{1} \neq \frac{1}{2}$ :

$$
\frac{\varepsilon}{2 \pi} P_{(j, 0)}^{-}=j_{1}+\underset{k_{1} \in \mathbb{Z}}{\operatorname{argmin}}\left|k_{1}+j_{1}\right|^{2}=\left\{\begin{array}{ll}
j_{1} & \text { for } j_{1} \in\left[0, \frac{1}{2}\right) \\
j_{1}-1 & \text { for } j_{1} \in\left(\frac{1}{2}, 1\right]
\end{array} .\right.
$$

The wave $U_{(j, 0)}^{-}$is right-going in the sense of Definition 3.1 if and only if $j_{1} \in\left[0, \frac{1}{2}\right)$. 
Remark 3.7 (Outgoing wave condition in a homogeneous medium). Let $u_{R}^{-}$be as in Definition 3.2. Remark 3.6 implies that, for every index $\lambda=(j, m) \in I_{R}$, there exists an index $k=k(\lambda) \in \mathbb{Z}^{2}$ with $U_{\lambda}^{-}(x)=e^{2 \pi i(k+j) \cdot x / \varepsilon}=e^{2 \pi i R(k+j) \cdot x / R \varepsilon}$. Using the shorthand notation $l(\lambda):=R(k(\lambda)+j) \in \mathbb{Z}^{2}$, the Bloch expansion of $u_{R}^{-}$can be rewritten as a Fourier expansion,

$$
u_{R}^{-}(x)=\sum_{\lambda \in I_{R}} \alpha_{\lambda, R}^{-} U_{\lambda}^{-}(x)=\sum_{\lambda \in I_{R}} \alpha_{\lambda, R}^{-} e^{2 \pi i l(\lambda) \cdot x / R \varepsilon} .
$$

By Remark 3.6, the basis function $U_{\lambda}^{-}(x)$ is right-going in the sense of Definition 3.1 if and only if $l_{1}(\lambda)>0$. This simplifies the radiation condition: The function $u$ with the truncations $u_{R}^{-}$on the left satisfies the outgoing wave condition (3.7) if and only if

$$
\sum_{\substack{\lambda \in I_{R} \\ l_{1}(\lambda)>0}}\left|\alpha_{\lambda, R}^{-}\right|^{2} \rightarrow 0 \quad \text { as } R \rightarrow \infty .
$$

We emphasize that, in order to evaluate the radiation condition, the map $l: I_{R} \rightarrow \mathbb{Z}^{2}$ need not be evaluated (we know that it is bijective). By expanding $u_{R}^{-}$in a classical Fourier series, $u_{R}^{-}(x)=\sum_{l \in \mathbb{Z}^{2}} \beta_{l, R}^{-} e^{2 \pi i l \cdot x / R \varepsilon}$ with some coefficients $\beta_{l, R}^{-}$, the outgoing wave condition $(3.18)$ is equivalent to $\sum_{\substack{l \in \mathbb{Z}^{2} \\ l_{1}>0}}\left|\beta_{l, R}^{-}\right|^{2} \rightarrow 0$ as $R \rightarrow \infty$.

\section{Comparison to the outgoing wave condition of Fliss and Joly [16]}

We claim that the outgoing wave condition of Fliss and Joly is formally stronger than our condition. More precisely: Every solution $u$ that satisfies the radiation condition (1.5) of [16] satisfies also our radiation condition (3.6) (we restrict the considerations here to the right side $\left.x_{1} \rightarrow+\infty\right)$. Indeed, let $u$ be as in (1.5), i.e. a finite sum of right-going Bloch-waves plus an exponentially decaying remainder $w^{+}(x)$. In order to check (3.6) for $u$, it suffices to verify, for each of the finitely many terms, the smallness of its $\Pi_{<0}^{+}$-projection. The smallness of the projection of $w^{+}(x)$ is clear because of the exponential decay of $w^{+}(x)$ and the boundedness of the projection operator. The smallness of the projection for each of the Bloch waves is shown in the subsequent lemma.

Since the expansion (1.5) contains only vertically periodic waves with frequency $\omega$ that are outgoing, we restrict our analysis to such Bloch-waves $U_{\lambda}^{+}$.

Lemma 3.8 (Right-going Bloch waves satisfy the radiation condition). Let $K \in \mathbb{N}$ denote the number of periodicity cells in vertical direction and let $U_{\lambda}^{+}$be a Bloch wave with $\lambda=(j, m) \in Z \times \mathbb{N}_{0}, j_{2} \in Q_{K}=\left\{0, \frac{1}{K}, \ldots, \frac{K-1}{K}\right\}, \mu_{m}^{+}(j)=\omega^{2}$, and $P_{\lambda}^{+}>0$. We impose that the frequency $\omega$ satisfies Assumption 1.1 (hence $m=0$ ). Then, as $\mathbb{N} K \ni R \rightarrow \infty:$

$$
\begin{aligned}
& f_{R Y_{\varepsilon}}\left|\Pi_{<0}^{+}\left(\left(U_{\lambda}^{+}\right)_{R}^{+}\right)\right|^{2} \rightarrow 0, \\
& f_{R Y_{\varepsilon}}\left|\Pi_{\geq 0}^{+}\left(\left(U_{\lambda}^{+}\right)_{R}^{+}\right)\right|^{2} \rightarrow 1 .
\end{aligned}
$$


Proof. Step 1: Equivalence of (3.19) and (3.20). By $L^{2}$-orthogonality of the projections we can calculate

$$
1=f_{R Y_{\varepsilon}}\left|\left(U_{\lambda}^{+}\right)_{R}^{+}\right|^{2}=f_{R Y_{\varepsilon}}\left|\Pi_{\geq 0}^{+}\left(\left(U_{\lambda}^{+}\right)_{R}^{+}\right)\right|^{2}+f_{R Y_{\varepsilon}}\left|\Pi_{<0}^{+}\left(\left(U_{\lambda}^{+}\right)_{R}^{+}\right)\right|^{2},
$$

which yields the equivalence of 3.19 and 3.20 .

Step 2: Proof of $(3.19)$. Let $\lambda=(j, m)=(j, 0)$ be as in the lemma. Arguing exactly as in Lemma 3.5 we conclude that contributions from energy levels $m \geq 1$ are negligible,

$$
f_{R Y_{\varepsilon}}\left|\Pi_{m \geq 1}^{\mathrm{ev},+}\left(\left(U_{\lambda}^{+}\right)_{R}^{+}\right)\right|^{2} \rightarrow 0 \quad \text { as } R \rightarrow \infty
$$

Consequently, for the weighted $L^{2}$-norm of $\Pi_{<0}^{+}\left(\left(U_{\lambda}^{+}\right)_{R}^{+}\right)$we calculate

$$
\begin{aligned}
f_{R Y_{\varepsilon}}\left|\Pi_{<0}^{+}\left(\left(U_{\lambda}^{+}\right)_{R}^{+}\right)\right|^{2} & =\sum_{\substack{\tilde{\lambda} \in I_{R} \cap I_{<0}^{+}\\
}}\left|\left\langle\left(U_{\lambda}^{+}\right)_{R}^{+}, U_{\tilde{\lambda}}\right\rangle_{R}\right|^{2}=\sum_{\substack{\tilde{\lambda} \in I_{R} \cap I_{<0}^{+} \\
\tilde{m}=0}}\left|\left\langle\left(U_{\lambda}^{+}\right)_{R}^{+}, U_{\tilde{\lambda}}\right\rangle_{R}\right|^{2}+o(1) \\
& =\sum_{\substack{\tilde{\lambda} \in I_{R} \cap I_{<0}^{+} \\
\tilde{m}=0, \tilde{j}_{2}=j_{2}}}\left|\left\langle\left(U_{\lambda}^{+}\right)_{R}^{+}, U_{\tilde{\lambda}}\right\rangle_{R}\right|^{2}+o(1) \quad \text { as } R \rightarrow \infty .
\end{aligned}
$$

In the last line we exploited that due to $j_{2} \in Q_{R}$ all scalar products with $\tilde{j}_{2} \neq j_{2}$ vanish. Next we show that there exists a constant $C=C(\lambda)>0$ such that

$$
\left|\left\langle\left(U_{\lambda}^{+}\right)_{R}^{+}, U_{\tilde{\lambda}}\right\rangle_{R}\right| \leq \frac{C}{R}
$$

for all $\tilde{\lambda}=(\tilde{j}, 0) \in I_{R} \cap I_{<0}^{+}$with $\tilde{j}_{2}=j_{2}$. Indeed, a direct calculation analogous to that of Lemma A.1 yields

$$
\left\langle\left(U_{\lambda}^{+}\right)_{R}^{+}, U_{\tilde{\lambda}}\right\rangle_{R}=e^{-2 \pi i j_{1} R} \frac{C(\lambda, \tilde{\lambda})}{R} \frac{1-e^{2 \pi i\left(\tilde{j}_{1}-j_{1}\right) R}}{1-e^{2 \pi i\left(\tilde{j}_{1}-j_{1}\right)}}
$$

with $C(\lambda, \tilde{\lambda}):=f_{(0, \varepsilon)^{2}} \Psi_{\lambda}^{+} \bar{\Psi}_{\tilde{\lambda}}^{+} e^{2 \pi i\left(\tilde{j}_{1}-j_{1}\right) y_{1} / \varepsilon} d y$. In particular $|C(\lambda, \tilde{\lambda})| \leq 1$ and therefore

$$
\left|\left\langle\left(U_{\lambda}^{+}\right)_{R}^{+}, U_{\tilde{\lambda}}\right\rangle_{R}\right| \leq \frac{1}{R}\left|\frac{1-e^{2 \pi i\left(\tilde{j}_{1}-j_{1}\right) R}}{1-e^{2 \pi i\left(\tilde{j}_{1}-j_{1}\right)}}\right| \leq \frac{1}{R} \frac{2}{\left|1-e^{2 \pi i\left(\tilde{j}_{1}-j_{1}\right)}\right|} .
$$

We now exploit $P_{\lambda}^{+}>0$. The eigenvalue $\mu_{0}^{+}(j)$ is simple, hence the Poynting number $P_{\lambda}^{+}=P_{(j, 0)}^{+}$is continuous in the wave number $j$. One thus finds a positive constant $\delta=\delta(\lambda)>0$ such that

$$
\left|1-e^{2 \pi i\left(\tilde{j}_{1}-j_{1}\right)}\right|>\delta
$$

for all $\tilde{\lambda}=(\tilde{j}, 0) \in I_{R} \cap I_{<0}^{+}$with $\tilde{j}_{2}=j_{2}$. Together with 3.22 this yields the claim 3.21 with $C=\frac{2}{\delta}$. 
With estimate (3.21) at hand we conclude

$$
\begin{aligned}
f_{R Y_{\varepsilon}}\left|\Pi_{<0}^{+}\left(\left(U_{\lambda}^{+}\right)_{R}^{+}\right)\right|^{2} & \leq \frac{C^{2}}{R^{2}} \mid\left\{\tilde{\lambda}=(\tilde{j}, 0) \in I_{R} \cap I_{<0}^{+} \text {with } \tilde{j}_{2}=j_{2}\right\} \mid+o(1) \\
& \leq \frac{C^{2}}{R}+o(1) \rightarrow 0 \quad \text { as } R \rightarrow \infty
\end{aligned}
$$

which was the claim.

\section{The sesquilinear form $b_{R}^{ \pm}$}

Let $\eta=\eta_{R}$ be a family of cut-off functions as in 3.10). In view of Relation (3.13) of Lemma 3.4. the outgoing wave conditions (3.6) and (3.7) are equivalent to outgoing wave conditions for the truncated functions $u_{R, \eta}^{ \pm}:=u_{R}^{ \pm} \eta$. More precisely, they are equivalent to the conditions

$$
f_{W_{R}}\left|\Pi_{<0}^{+}\left(u_{R, \eta}^{+}\right)\right|^{2} \rightarrow 0 \quad \text { and } \quad f_{W_{R}} \mid\left(\left.\Pi_{>0}^{-}\left(u_{R, \eta}^{-}\right)\right|^{2} \rightarrow 0 \quad \text { as } \quad R \rightarrow \infty .\right.
$$

In the proof of our uniqueness result we will use (3.23) instead of the original conditions (3.6) and (3.7). In fact, even a weaker form of the conditions is sufficient and we discuss this relaxation in the following.

Corresponding to the energy flux definition in (3.1), we associate to a function $w \in H^{1}\left(W_{R} ; \mathbb{C}\right)$ on $W_{R}=R Y_{\varepsilon}$ the Poynting number

$$
B_{R}^{+}(w):=\operatorname{Im} f_{W_{R}} \bar{w}(x) e_{1} \cdot\left[a^{\varepsilon}(x) \nabla w(x)\right] d x .
$$

The quadratic expression $B_{R}^{-}$is defined analogously, with $a^{\varepsilon}(x)$ replaced by 1 .

Definition 3.9 (Weaker form of the outgoing wave condition). For $K, R \in \mathbb{N}$ with $R \in K \mathbb{N}$ we consider $u \in H_{\text {loc }}^{1}(\mathbb{R} \times(0, \varepsilon K) ; \mathbb{C})$ and $u_{R, \eta}^{ \pm}$as in 3.23 . We say that $u$ satisfies the energetic outgoing wave condition on the right, if

$$
B_{R}^{+}\left(\Pi_{<0}^{+} \Pi_{m=0}^{\mathrm{ev},+}\left(u_{R, \eta}^{+}\right)\right) \rightarrow 0 \text { as } R \rightarrow \infty .
$$

Accordingly, we say that u satisfies the energetic outgoing wave condition on the left, if

$$
B_{R}^{-}\left(\Pi_{>0}^{-} \Pi_{m=0}^{\mathrm{ev},-}\left(u_{R, \eta}^{-}\right)\right) \rightarrow 0 \text { as } R \rightarrow \infty .
$$

In two respects, the condition (3.25) is similar to the condition (3.23): the function $u$ is considered at the far right since only $u_{R, \eta}^{+}$is used. In view of Lemma 3.5, contributions from energy levels $m \geq 1$ can be neglected and we consider only $\prod_{m=0}^{\mathrm{ev},+}\left(u_{R, \eta}^{+}\right)$. Furthermore, this function is projected to left-going waves, i.e. only $\Pi_{<0}^{+} \Pi_{m=0}^{\mathrm{ev},+}\left(u_{R, \eta}^{+}\right)$is studied. The main difference between the two conditions is that, instead of looking at the weighted $L^{2}$-norm, one demands in (3.25) a decay property for the energy-flux quantity $B_{R}^{+}$. At the end of this section, we will see that condition 3.23 (together with the uniform $L^{2}$-bounds and the solution property) implies (3.25). 
The definition of $B_{R}^{+}$in (3.24) suggests to introduce additionally the (nonsymmetric) sesquilinear forms $b_{R}^{ \pm}: L^{2}\left(W_{R} ; \mathbb{C}\right) \times H^{1}\left(W_{R} ; \mathbb{C}\right) \rightarrow \mathbb{C}$,

$$
\begin{aligned}
b_{R}^{+}(u, v) & :=\int_{W_{R}} \bar{u}(x) e_{1} \cdot\left[a^{\varepsilon}(x) \nabla v(x)\right] d x, \\
b_{R}^{-}(u, v) & :=f_{W_{R}} \bar{u}(x) e_{1} \cdot \nabla v(x) d x .
\end{aligned}
$$

The definition is tailored to calculate energy fluxes. The energy flux of the left-going contributions of $u_{R, \eta}^{+}$(in the right half-plane) is quantified by

$$
\begin{aligned}
B_{R}^{+}\left(\Pi_{<0}^{+} u_{R, \eta}^{+}\right) & =\operatorname{Im} b_{R}^{+}\left(\Pi_{<0}^{+} u_{R, \eta}^{+}, \Pi_{<0}^{+} u_{R, \eta}^{+}\right) \\
& =\operatorname{Im} \int_{W_{R}} \overline{\Pi_{<0}^{+} u_{R, \eta}^{+}(x)} e_{1} \cdot\left[a^{\varepsilon}(x) \nabla\left(\Pi_{<0}^{+} u_{R, \eta}^{+}\right)(x)\right] d x .
\end{aligned}
$$

The connection to $P_{\lambda}^{ \pm}$is given by

$$
P_{\lambda}^{ \pm}=B_{R}^{ \pm}\left(U_{\lambda}^{ \pm}\right)=\operatorname{Im} b_{R}^{ \pm}\left(U_{\lambda}^{ \pm}, U_{\lambda}^{ \pm}\right) .
$$

Let us collect some properties of the sesquilinear forms $b_{R}^{ \pm}$.

Lemma 3.10 (Properties of the sesquilinear form $b_{R}^{ \pm}$). For $R \in \mathbb{N}$, the following holds:

1. Orthogonality property of $b_{R}^{ \pm}$. Let $\lambda, \tilde{\lambda} \in I_{R}$ be such that $\lambda=(j, m), \tilde{\lambda}=(\tilde{j}, \tilde{m})$ with $j \neq \tilde{j}$. Then $U_{\lambda}^{ \pm}, U_{\tilde{\lambda}}^{ \pm}$of 2.8 satisfy

$$
b_{R}^{ \pm}\left(U_{\lambda}^{ \pm}, U_{\tilde{\lambda}}^{ \pm}\right)=0
$$

2. Convergence property of $b_{R}^{ \pm}$. Let sequences $u_{R} \in L^{2}\left(W_{R} ; \mathbb{C}\right)$ and $v_{R} \in H^{1}\left(W_{R} ; \mathbb{C}\right)$ be such that

$$
f_{W_{R}}\left|u_{R}\right|^{2}+\left|\nabla v_{R}\right|^{2} \leq C_{0}
$$

with $C_{0}$ independent of $R$. Let either $f_{W_{R}}\left|u_{R}\right|^{2} \rightarrow 0$ or $f_{W_{R}}\left|\nabla v_{R}\right|^{2} \rightarrow 0$ as $R \rightarrow \infty$. Then there holds

$$
b_{R}^{ \pm}\left(u_{R}, v_{R}\right) \rightarrow 0 .
$$

Proof. 1. We prove 3.29 for $U_{\lambda}^{+}, U_{\tilde{\lambda}}^{+}$, the argument for $U_{\lambda}^{-}, U_{\tilde{\lambda}}^{-}$is analogous. We have to show that

$$
b_{R}^{+}\left(U_{\lambda}^{+}, U_{\tilde{\lambda}}^{+}\right)=f_{W_{R}} \overline{U_{\lambda}^{+}}(x) e_{1} \cdot\left[a^{\varepsilon}(x) \nabla U_{\tilde{\lambda}}^{+}(x)\right] d x \stackrel{!}{=} 0 .
$$

By definition of $U_{\lambda}^{+}$and $U_{\tilde{\lambda}}^{+}$there holds

$$
\begin{aligned}
\overline{U_{\lambda}^{+}}(x) & =\overline{\Psi_{\lambda}^{+}}(x) e^{-i 2 \pi j \cdot x / \varepsilon} \\
\nabla U_{\tilde{\lambda}}^{+}(x) & =\left[\nabla \Psi_{\tilde{\lambda}}^{+}(x)+(i 2 \pi \tilde{j} / \varepsilon) \Psi_{\tilde{\lambda}}^{+}(x)\right] e^{i 2 \pi \tilde{j} \cdot x / \varepsilon}
\end{aligned}
$$


with $\varepsilon$-periodic functions $\Psi_{\lambda}^{+}, \Psi_{\tilde{\lambda}}^{+}$, and $\nabla \Psi_{\tilde{\lambda}}^{+}$. Due to the $\varepsilon$-periodicity of $a^{\varepsilon}$ and since $j, \tilde{j} \in Q_{R}$ satisfy $j \neq \tilde{j}$, we can apply Lemma A.1 of the appendix, which yields the claim.

2. We show the claim for $b_{R}^{+}$, the argument for $b_{R}^{-}$is analogous. The Cauchy-Schwarz inequality allows to calculate

$$
\begin{aligned}
& \left|b_{R}^{+}\left(u_{R}, v_{R}\right)\right|=\left|f_{W_{R}} \bar{u}_{R}(x) e_{1} \cdot\left[a^{\varepsilon}(x) \nabla v_{R}(x)\right] d x\right| \\
& \quad \leq\left\|a^{\varepsilon}\right\|_{\infty}\left(f_{W_{R}}\left|u_{R}\right|^{2}\right)^{1 / 2}\left(f_{W_{R}}\left|\nabla v_{R}\right|^{2}\right)^{1 / 2} \rightarrow 0 \quad \text { as } R \rightarrow \infty,
\end{aligned}
$$

which concludes the proof.

Lemma 3.10 shows that the outgoing wave condition $(3.23)$ together with the $L^{2}$ bounds of Definition 3.3 imply (3.25). Indeed, by (3.23), there holds

$$
f_{W_{R}}\left|\Pi_{<0}^{+} \Pi_{m=0}^{\mathrm{ev},+}\left(u_{R, \eta}^{+}\right)\right|^{2} \leq f_{W_{R}}\left|\Pi_{<0}^{+}\left(u_{R, \eta}^{+}\right)\right|^{2} \rightarrow 0 \text { as } R \rightarrow \infty .
$$

Moreover, $\Pi_{<0}^{+} \Pi_{m=0}^{\mathrm{ev},+}\left(u_{R, \eta}^{+}\right)$satisfies $f_{W_{R}}\left|\nabla\left(\Pi_{<0}^{+} \Pi_{m=0}^{\mathrm{ev},+}\left(u_{R, \eta}^{+}\right)\right)\right|^{2} \leq C$ with $C$ independent of $R$ due to Lemma A.3 and Lemma A.4 of the appendix. Lemma 3.10 provides

$$
B_{R}^{+}\left(\Pi_{<0}^{+} \Pi_{m=0}^{\mathrm{ev},+}\left(u_{R, \eta}^{+}\right)\right)=\operatorname{Im} b_{R}^{+}\left(\Pi_{<0}^{+} \Pi_{m=0}^{\mathrm{ev},+}\left(u_{R, \eta}^{+}\right), \Pi_{<0}^{+} \Pi_{m=0}^{\mathrm{ev},+}\left(u_{R, \eta}^{+}\right)\right) \rightarrow 0 \text { as } R \rightarrow \infty,
$$

and hence (3.25).

Remark 3.11 (On the sesquilinear form $b_{R}^{ \pm}$). Another choice of a bilinear form is

$$
\tilde{b}_{R}^{+}(u, v):=\frac{1}{2} f_{W_{R}}\left\{\bar{u}(x) e_{1} \cdot\left[a^{\varepsilon}(x) \nabla v(x)\right]-v(x) e_{1} \cdot\left[a^{\varepsilon}(x) \nabla \bar{u}(x)\right]\right\} d x .
$$

With this choice, the energy flux $B_{R}^{ \pm}$can be calculated as before, since $\operatorname{Im} b_{R}^{+}(u, u)=$ $\operatorname{Im} \tilde{b}_{R}^{+}(u, u)$ holds for every $u$. The properties of Lemma 3.10 remain also unchanged, the only additional requirement would be an $H^{1}$-bound also for $u_{R}$ in (3.30).

The advantage of $\tilde{b}_{R}^{+}(u, v)$ is that more orthogonality can be expected for $\tilde{b}_{R}^{+}$than for $b_{R}^{+}$. Essentially, the bilinear form $q$ of (27) in [16] coincides with $\tilde{b}_{R}^{+}$(up to a factor 2 , our coefficient $a^{\varepsilon}$, and the fact that we use a volume integral for the averaging). In Theorem 3 of [16], an orthogonality property is shown for $q$, which resembles our orthogonality relation (3.29), stating that orthogonality holds also for $\lambda=(j, m)$ and $\tilde{\lambda}=(j, \tilde{m})$ with $m \neq \tilde{m}$. Unfortunately, such an orthogonality is only true for basis functions corresponding to the same frequency $\omega$, while our analysis of an interface would require orthogonality independent of the frequency.

\section{Bloch measures and uniqueness properties}

Our aim is to show uniqueness properties of the transmision Problem 1.2 with incoming wave $U_{\text {inc }}$ and outgoing wave conditions. Following the standard procedure of uniqueness proofs, we consider two solutions $u$ and $\tilde{u}$ of the problem. Due to linearity of the 
system, the difference $v:=u-\tilde{u}$ satisfies again (1.1). Furthermore, it satisfies outgoing wave conditions on the left and on the right according to Definition 3.3, without any incoming wave $U_{\text {inc }}$. At this point, we have exploited the triangle inequality: Certain projections of $u$ and $\tilde{u}$ tend to zero in a weighted $L^{2}$-norm, hence also the projections of $v$ tend to zero. We can not show that $v$ vanishes (indeed, as explained in the introduction, we expect that there exist nontrivial solutions for vanishing $U_{\text {inc }}$ ). But we can show that the functions $v_{R}^{ \pm}$consist, in the limit $R \rightarrow \infty$, only of vertical waves. The right object to study is the Bloch measure associated with $v_{R}^{ \pm}$.

We recall that the frequency assumption (1.7) implies that, in the limit $R \rightarrow \infty$, the discrete Bloch expansions of $u_{R}^{ \pm}$contain only modes corresponding to $\lambda=(j, m)$ with $m=0$, see Lemma 3.5 .

\subsection{Bloch measures}

In the definition of Bloch measures we use the space of all Radon measures on the unit square $Z$, which we denote as $\mathcal{M}(Z)$. It is the dual of the space of continuous functions on $Z$ and accordingly equipped with the topology of weak-* convergence. This means, in particular, that every bounded sequence in $\mathcal{M}(Z)$ has a convergent subsequence.

Definition 4.1 (Discrete Bloch measure). Let $u_{R} \in L^{2}\left(W_{R} ; \mathbb{C}\right)$ be a sequence of functions with discrete Bloch-expansions

$$
u_{R}(x)=\sum_{\lambda \in I_{R}} \alpha_{\lambda}^{ \pm} U_{\lambda}^{ \pm}(x),
$$

where $\alpha_{\lambda}^{ \pm}=\alpha_{\lambda}^{ \pm}(R)$ depend on $R \in \mathbb{N}$. Given these coefficients, for fixed $l \in \mathbb{N}_{0}$, we define the l-th discrete Bloch-measure $\nu_{l, R}^{ \pm} \in \mathcal{M}(Z)$ by

$$
\nu_{l, R}^{ \pm}:=\sum_{\lambda=(j, l) \in I_{R}}\left|\alpha_{\lambda}^{ \pm}\right|^{2} \delta_{j},
$$

where $\delta_{j}$ denotes the Dirac measure at the frequency $j \in Z$.

For $u_{R}$ fixed, $\nu_{l, R}^{ \pm}$is a non-negative Radon measure on $Z=[0,1]^{2}$. There holds

$$
\sum_{l=0}^{\infty} \int_{Z} d \nu_{l, R}^{ \pm}=\sum_{\lambda \in I_{R}}\left|\alpha_{\lambda}^{ \pm}\right|^{2}=f_{W_{R}}\left|u_{R}\right|^{2} .
$$

Our aim is to study the limiting behavior $R \rightarrow \infty$ of the discrete Bloch measures $\nu_{l, R}^{ \pm}$.

Definition 4.2 (Bloch measure). For $\varepsilon>0, K \in \mathbb{N}$ and $h=K \varepsilon$, let $u$ be a function $u \in L_{\text {loc }}^{2}(\mathbb{R} \times(0, h) ; \mathbb{C})$. We consider a sequence $\mathbb{N} K \ni R \rightarrow \infty$. We extract $u_{R, \eta}^{ \pm}:=u_{R}^{ \pm} \eta$ according to Definition 3.2 with a sequence of cut-off functions $\eta=\eta_{R}$ as in (3.10). For $l \in \mathbb{N}_{0}$, let $\nu_{l, R}^{ \pm}$be the discrete Bloch measures associated with $u_{R, \eta}^{ \pm}$.

We say that the measure $\nu_{l, \infty}^{ \pm} \in \mathcal{M}(Z)$ is a Bloch measure generated by $u$ if there holds, along a subsequence $R \rightarrow \infty$, in the sense of measures (i.e. weak-*),

$$
\nu_{l, R}^{ \pm} \rightarrow \nu_{l, \infty}^{ \pm}
$$


Relation (4.3) is equivalent to the following: for every test-function $\phi \in C(Z)$ on $Z=[0,1]^{2}$ there holds

$$
\sum_{\lambda=(j, l) \in I_{R}} \phi(j)\left|\alpha_{\lambda}^{ \pm}\right|^{2}=\int_{Z} \phi d \nu_{l, R}^{ \pm} \rightarrow \int_{Z} \phi d \nu_{l, \infty}^{ \pm} \quad \text { as } R \rightarrow \infty .
$$

The methods of Section 4.3 force us to work with cut-off functions, i.e. with $u_{R, \eta}^{ \pm}$and with $\nu_{l, R}^{ \pm}$. Nevertheless, one may also study the discrete Bloch measures $\tilde{\nu}_{l, R}^{ \pm}$associated to $u_{R}^{ \pm}$(without cut-off function). In the limit $R \rightarrow \infty$, the two measures coincide,

$$
\nu_{l, R}^{ \pm}-\tilde{\nu}_{l, R}^{ \pm} \rightarrow 0
$$

in the sense of measures. In particular, the Bloch measure generated by $u_{R, \eta}^{ \pm}$is independent of the choice of $\eta$.

\subsection{Energy estimates and consequences for the Bloch measure}

Up to this point (with the exception of Lemma 3.5), our considerations have been completely abstract in the following sense: Given a function $u \in L_{\text {loc }}^{2}(\mathbb{R} \times(0, h) ; \mathbb{C})$, we have constructed restrictions of $u$ to large boxes, projections of these restrictions, and finally discrete and limiting Bloch measures corresponding to $u$. Except for regularity properties, we have not exploited the Helmholtz equation. In this section, we will derive relations that express a physical law: energy conservation. This will eventually lead us to the uniqueness properties which are expressed with the Bloch measures.

The subsequent result states that, while left-going waves on the right vanish by the outgoing wave condition, right-going waves vanish by energy conservation.

Proposition 4.3. Let Assumption 1.1 on $\omega>0$ be satisfied and let $v$ be a solution to the scattering problem (1.1), periodic in vertical direction, satisfying outgoing wave conditions on the left and on the right according to Definition 3.3, without incoming wave, i.e. $U_{\mathrm{inc}} \equiv 0$. For a sequence of cut-off functions $\eta=\eta_{R}$ as in (3.10) we consider $v_{R, \eta}^{ \pm}:=v_{R}^{ \pm} \eta_{R}=\sum_{\lambda \in I_{R}} \alpha_{\lambda, R}^{ \pm} U_{\lambda}^{ \pm}$, c.f. Definition 3.2. Then, as $\mathbb{N} K \ni R \rightarrow \infty$,

$$
\sum_{\substack{\lambda=(j, 0) \\ \lambda \in I_{R} \cap I_{\leq 0}^{-}}}\left|\alpha_{\lambda, R}^{-}\right|^{2} P_{\lambda}^{-} \rightarrow 0 \quad \text { and } \sum_{\substack{\lambda=(j, 0) \\ \lambda \in I_{R} \cap I_{\geq 0}^{+}}}\left|\alpha_{\lambda, R}^{+}\right|^{2} P_{\lambda}^{+} \rightarrow 0
$$

Proof. Step 1: Energy flux equality. For $h=\varepsilon K$ and $R \in \mathbb{N} K$, we consider the special cut-off function $\vartheta(x)=\vartheta_{R}(x)$, defined for $x=\left(x_{1}, x_{2}\right)$ as

$$
\vartheta(x):= \begin{cases}1 & \text { if }\left|x_{1}\right| \leq \varepsilon R \\ 2-\frac{\left|x_{1}\right|}{\varepsilon R} & \text { if } \varepsilon R<\left|x_{1}\right|<2 \varepsilon R \\ 0 & \text { if }\left|x_{1}\right| \geq 2 \varepsilon R\end{cases}
$$

We multiply the Helmholtz equation (1.1) with coefficients $a=a^{\varepsilon}$ and solution $v$ by the test-function $\vartheta(x) \bar{v}(x)$. An integration over $\mathbb{R} \times(0, h)$ and integration by parts yields 
(no boundary terms appear due to periodicity in $x_{2}$-direction and compact support):

$$
\int_{\mathbb{R}} \int_{0}^{h}\left\{a^{\varepsilon} \vartheta|\nabla v|^{2}+a^{\varepsilon} \partial_{x_{1}} \vartheta \bar{v} \partial_{x_{1}} v\right\}=\omega^{2} \int_{\mathbb{R}} \int_{0}^{h} \vartheta|v|^{2} .
$$

Due to the special choice of $\vartheta$ and $a^{\varepsilon}(x)=1$ for $x_{1}<0$, this equation reads

$$
f_{-2 R \varepsilon}^{-R \varepsilon} \int_{0}^{h} \bar{v} \partial_{x_{1}} v-f_{R \varepsilon}^{2 R \varepsilon} \int_{0}^{h} \bar{v} a^{\varepsilon} \partial_{x_{1}} v=\int_{\mathbb{R}} \int_{0}^{h}\left\{\omega^{2} \vartheta|v|^{2}-a^{\varepsilon} \vartheta|\nabla v|^{2}\right\}
$$

On the left-hand side, we recognize the sesquilinear forms $b_{R}^{ \pm}$of (3.27). Because of periodicity in $x_{2}$-direction, we may write

$$
h\left[b_{R}^{-}\left(v_{R}^{-}, v_{R}^{-}\right)-b_{R}^{+}\left(v_{R}^{+}, v_{R}^{+}\right)\right]=\int_{\mathbb{R}} \int_{0}^{h}\left\{\omega^{2} \vartheta|v|^{2}-a^{\varepsilon} \vartheta|\nabla v|^{2}\right\} .
$$

Since the right hand side is real, taking the imaginary part of 4.5 yields

$$
\operatorname{Im} b_{R}^{-}\left(v_{R}^{-}, v_{R}^{-}\right)-\operatorname{Im} b_{R}^{+}\left(v_{R}^{+}, v_{R}^{+}\right)=0 .
$$

Relation 4.6 is an energy conservation: The energy flux into the domain from the left must coincide with the energy flux out of the domain at the right.

Step 2: Truncations and $(m \geq 1)$-waves. We start this part of the proof with an observation regarding the cut-off functions; we want to have them in the argument of the sesquilinear form. Due to Lemma A.3 and the properties of the cut-off functions $\eta=\eta_{R}$ we have

$$
f_{W_{R}}\left|v_{R}^{ \pm}-v_{R, \eta}^{ \pm}\right|^{2}+\left|\nabla v_{R}^{ \pm}-\nabla v_{R, \eta}^{ \pm}\right|^{2} \leq \frac{C}{R},
$$

and therefore, by Lemma 3.10 ,

$$
\begin{aligned}
& b_{R}^{ \pm}\left(v_{R}^{ \pm}, v_{R}^{ \pm}\right)-b_{R}^{ \pm}\left(v_{R, \eta}^{ \pm}, v_{R, \eta}^{ \pm}\right) \\
& \quad=b_{R}^{ \pm}\left(v_{R}^{ \pm}-v_{R, \eta}^{ \pm}, v_{R}^{ \pm}\right)+b_{R}^{ \pm}\left(v_{R, \eta}^{ \pm}, v_{R}^{ \pm}-v_{R, \eta}^{ \pm}\right) \rightarrow 0 \quad \text { as } R \rightarrow \infty .
\end{aligned}
$$

The energy conservation 4.6 therefore implies that, as $R \rightarrow \infty$,

$$
\operatorname{Im} b_{R}^{-}\left(v_{R, \eta}^{-}, v_{R, \eta}^{-}\right)-\operatorname{Im} b_{R}^{+}\left(v_{R, \eta}^{+}, v_{R, \eta}^{+}\right) \rightarrow 0 .
$$

We next decompose the sesquilinear forms $b_{R}^{ \pm}$according to the projections of Definition 3.1, and suppress the superscript " \pm " in the projection. We exploit sesquilinearity of $b_{R}^{+}$in both arguments and write

$$
\begin{aligned}
& \operatorname{Im} b_{R}^{+}\left(v_{R, \eta}^{+}, v_{R, \eta}^{+}\right) \\
& =\operatorname{Im} b_{R}^{+}\left(\Pi_{m \geq 1}^{\mathrm{ev}}\left(v_{R, \eta}^{+}\right), v_{R, \eta}^{+}\right)+\operatorname{Im} b_{R}^{+}\left(\Pi_{m=0}^{\mathrm{ev}}\left(v_{R, \eta}^{+}\right), \Pi_{m \geq 1}^{\mathrm{ev}}\left(v_{R, \eta}^{+}\right)\right) \\
& \quad+\operatorname{Im} b_{R}^{+}\left(\Pi_{m=0}^{\mathrm{ev}}\left(v_{R, \eta}^{+}\right), \Pi_{m=0}^{\mathrm{ev}}\left(v_{R, \eta}^{+}\right)\right) .
\end{aligned}
$$


We want to exploit the smallness of $m \geq 1$-contributions of Lemma 3.5. The regularity result of Lemma A.3 together with the properties of the sesquilinear form $b_{R}^{+}$of Lemma 3.10 yield that the first term on the right hand side of 4.9 vanishes in the limit as $R \rightarrow \infty$. For the second term we apply Lemma A.4, which provides that also the gradient of $\Pi_{m \geq 1}^{\mathrm{ev}}\left(v_{R, \eta}^{+}\right)$is small; Lemma 3.10 implies

$$
b_{R}^{+}\left(\Pi_{m=0}^{\mathrm{ev}}\left(v_{R, \eta}^{+}\right), \Pi_{m \geq 1}^{\mathrm{ev}}\left(v_{R, \eta}^{+}\right)\right) \rightarrow 0 \quad \text { as } R \rightarrow \infty,
$$

i.e. also the second term on the right hand side of 4.9 vanishes in the limit. We find that, as $R \rightarrow \infty$,

$$
\operatorname{Im} b_{R}^{+}\left(v_{R, \eta}^{+}, v_{R, \eta}^{+}\right)=\operatorname{Im} b_{R}^{+}\left(\Pi_{m=0}^{\mathrm{ev}}\left(v_{R, \eta}^{+}\right), \Pi_{m=0}^{\mathrm{ev}}\left(v_{R, \eta}^{+}\right)\right)+o(1) .
$$

Step 3: Energy flux and outgoing wave conditions. In this step we decompose $\operatorname{Im} b_{R}^{+}\left(\Pi_{m=0}^{\mathrm{ev}}\left(v_{R, \eta}^{+}\right), \Pi_{m=0}^{\mathrm{ev}}\left(v_{R, \eta}^{+}\right)\right)$as follows:

$$
\begin{aligned}
\operatorname{Im} b_{R}^{+}\left(\Pi_{m=0}^{\mathrm{ev}}\left(v_{R, \eta}^{+}\right), \Pi_{m=0}^{\mathrm{ev}}\left(v_{R, \eta}^{+}\right)\right) \\
=\operatorname{Im} b_{R}^{+}\left(\Pi_{<0}^{+} \Pi_{m=0}^{\mathrm{ev}}\left(v_{R, \eta}^{+}\right), \Pi_{<0}^{+} \Pi_{m=0}^{\mathrm{ev}}\left(v_{R, \eta}^{+}\right)\right) \\
\quad+\operatorname{Im} b_{R}^{+}\left(\Pi_{<0}^{+} \Pi_{m=0}^{\mathrm{ev}}\left(v_{R, \eta}^{+}\right), \Pi_{\geq 0}^{+} \Pi_{m=0}^{\mathrm{ev}}\left(v_{R, \eta}^{+}\right)\right) \\
\quad+\operatorname{Im} b_{R}^{+}\left(\Pi_{\geq 0}^{+} \Pi_{m=0}^{\mathrm{ev}}\left(v_{R, \eta}^{+}\right), \Pi_{\geq 0}^{+} \Pi_{m=0}^{\mathrm{ev}}\left(v_{R, \eta}^{+}\right)\right) \\
\quad+\operatorname{Im} b_{R}^{+}\left(\Pi_{\geq 0}^{+} \Pi_{m=0}^{\mathrm{ev}}\left(v_{R, \eta}^{+}\right), \Pi_{<0}^{+} \Pi_{m=0}^{\mathrm{ev}}\left(v_{R, \eta}^{+}\right)\right) \\
=\operatorname{Im} b_{R}^{+}\left(\Pi_{<0}^{+} \Pi_{m=0}^{\mathrm{ev}}\left(v_{R, \eta}^{+}\right), \Pi_{<0}^{+} \Pi_{m=0}^{\mathrm{ev}}\left(v_{R, \eta}^{+}\right)\right) \\
\quad+\operatorname{Im} b_{R}^{+}\left(\Pi_{\geq 0}^{+} \Pi_{m=0}^{\mathrm{ev}}\left(v_{R, \eta}^{+}\right), \Pi_{\geq 0}^{+} \Pi_{m=0}^{\mathrm{ev}}\left(v_{R, \eta}^{+}\right)\right),
\end{aligned}
$$

where the last equality holds, since for $\lambda=(j, m=0) \in I_{<0}^{+}$and $\tilde{\lambda}=(\tilde{j}, m=0) \in I_{\geq 0}^{+}$ one always has $j \neq \tilde{j}$ and thus the mixed sesquilinear forms vanish due to orthogonality in the wave number, cf. Lemma 3.10. Exploiting the outgoing wave condition (3.23) on the right or, better, the weaker expression (3.25), we find that the first term on the right hand side of (4.11) vanishes in the limit $R \rightarrow \infty$. Hence

$$
\begin{aligned}
& \operatorname{Im} b_{R}^{+}\left(\Pi_{m=0}^{\mathrm{ev}}\left(v_{R, \eta}^{+}\right), \Pi_{m=0}^{\mathrm{ev}}\left(v_{R, \eta}^{+}\right)\right) \\
& \quad=\operatorname{Im} b_{R}^{+}\left(\Pi_{\geq 0}^{+} \Pi_{m=0}^{\mathrm{ev}}\left(v_{R, \eta}^{+}\right), \Pi_{\geq 0}^{+} \Pi_{m=0}^{\mathrm{ev}}\left(v_{R, \eta}^{+}\right)\right)+o(1) \quad \text { as } R \rightarrow \infty .
\end{aligned}
$$

We emphasize that we only used the energetic outgoing wave condition (3.25) in this calculation.

Combining (4.10) with 4.12) we finally obtain, as $R \rightarrow \infty$,

$$
\operatorname{Im} b_{R}^{+}\left(v_{R, \eta}^{+}, v_{R, \eta}^{+}\right)=\operatorname{Im} b_{R}^{+}\left(\Pi_{\geq 0}^{+} \Pi_{m=0}^{\mathrm{ev}}\left(v_{R, \eta}^{+}\right), \Pi_{\geq 0}^{+} \Pi_{m=0}^{\mathrm{ev}}\left(v_{R, \eta}^{+}\right)\right)+o(1) .
$$

Step 4: Consequences for outgoing waves. We analyze (4.13) further, exploiting the discrete Bloch expansion of $v_{R, \eta}^{ \pm}=\sum_{\lambda \in I_{R}} \alpha_{\lambda, R}^{ \pm} U_{\lambda}^{ \pm}$:

$$
\begin{aligned}
\operatorname{Im} & b_{R}^{+}\left(\Pi_{\geq 0}^{+} \Pi_{m=0}^{\mathrm{ev}}\left(v_{R, \eta}^{+}\right), \Pi_{\geq 0}^{+} \Pi_{m=0}^{\mathrm{ev}}\left(v_{R, \eta}^{+}\right)\right) \\
= & \operatorname{Im} \sum_{\lambda=(j, 0) \in I_{R} \cap I_{\geq 0}^{+}} \sum_{\tilde{\lambda}=(j, 0) \in I_{R} \cap I_{\geq 0}^{+}} \bar{\alpha}_{\lambda, R}^{+} \alpha_{\tilde{\lambda}, R}^{+} b_{R}^{+}\left(U_{\lambda}^{+}, U_{\tilde{\lambda}}^{+}\right) \\
= & \sum_{\lambda=(j, 0) \in I_{R} \cap I_{\geq 0}^{+}}\left|\alpha_{\lambda, R}^{+}\right|^{2} \operatorname{Im} b_{R}^{+}\left(U_{\lambda}^{+}, U_{\lambda}^{+}\right)=\sum_{\lambda=(j, 0) \in I_{R} \cap I_{\geq 0}^{+}}\left|\alpha_{\lambda, R}^{+}\right|^{2} P_{\lambda}^{+} .
\end{aligned}
$$


In the last line we again exploited the orthogonality of the sesquilinear form $b_{R}^{+}$in the wave number $j$, see Lemma 3.10, and the relation (3.28) for $P_{\lambda}^{ \pm}$. We may therefore write 4.13 as

$$
\operatorname{Im} b_{R}^{+}\left(v_{R, \eta}^{+}, v_{R, \eta}^{+}\right)=\sum_{\lambda=(j, 0) \in I_{R} \cap I_{\geq 0}^{+}}\left|\alpha_{\lambda, R}^{+}\right|^{2} P_{\lambda}^{+}+o(1) .
$$

On the left, we find similarly

$$
\operatorname{Im} b_{R}^{-}\left(v_{R, \eta}^{-}, v_{R, \eta}^{-}\right)=\sum_{\lambda=(j, 0) \in I_{R} \cap I_{\leq 0}^{-}}\left|\alpha_{\lambda, R}^{-}\right|^{2} P_{\lambda}^{-}+o(1) .
$$

The energy relation (4.6) together with the sign properties $P_{\lambda}^{+} \geq 0$ for $\lambda \in I_{\geq 0}^{+}$and $P_{\lambda}^{-} \leq 0$ for $\lambda \in I_{\leq 0}^{-}$allows to conclude 4.4.

\subsection{Proof of Theorem 1.3 and Theorem 1.5}

We study solutions to the Helmholtz equation (1.1). In order to prove Theorems 1.3 and 1.5, we have to check conditions that are satisfied by the support of Bloch measures of solutions. We recall the notation

$$
J_{=0, l}^{ \pm}=\left\{j \in Z=[0,1]^{2} \mid P_{\lambda}^{ \pm}=0 \text { for } \lambda=(j, l)\right\}
$$

for the index set corresponding to vertical waves.

Proof of Theorem 1.3. In the following, we consider solutions to the transmission Problem 1.2. We are interested in a function $v$ which is the difference of two solutions or, equivalently, a solution to the problem without incoming wave.

Proposition 4.4 (Solutions in absence of incoming waves). Let Assumption 1.1 on $\omega>0$ be satisfied and let $v$ be a solution to the scattering problem (1.1), periodic in vertical direction, satisfying outgoing wave conditions on the left and on the right according to Definition 3.3, without incoming wave. Let $\nu_{l, \infty}^{ \pm}$, with $l \in \mathbb{N}_{0}$, be Bloch measures that are generated by $v$. Then

$$
\begin{aligned}
\nu_{l, \infty}^{ \pm} & =0 \quad \text { for } l \geq 1, \\
\operatorname{supp}\left(\nu_{0, \infty}^{ \pm}\right) & \subset J_{=0,0}^{ \pm} .
\end{aligned}
$$

Proof. We only show the statement for the limiting Bloch measures $\nu_{l, \infty}^{+}$, the argument for $\nu_{l, \infty}^{-}$is analogous. Let $v_{R, \eta}^{+}=\sum_{\lambda \in I_{R}} \alpha_{\lambda, R}^{+} U_{\lambda}^{+}$be the expansion of the truncated solution. Then the corresponding discrete Bloch measures are given by

$$
\nu_{l, R}^{+}=\sum_{\lambda=(j, l) \in I_{R}}\left|\alpha_{\lambda, R}^{+}\right|^{2} \delta_{j} .
$$

The case $l \geq 1$ : From 3.15 we know that

$$
f_{W_{R}}\left|\Pi_{m \geq 1}^{\mathrm{ev}}\left(v_{R, \eta}^{+}\right)\right|^{2}=\sum_{\substack{\lambda=(j, m) \in I_{R} \\ m \geq 1}}\left|\alpha_{\lambda, R}^{+}\right|^{2} \leq \frac{C}{R},
$$


and hence

$$
\int_{Z} d \nu_{l, R}^{+}=\sum_{\lambda=(j, l) \in I_{R}}\left|\alpha_{\lambda, R}^{+}\right|^{2} \rightarrow 0 \quad \text { as } R \rightarrow \infty .
$$

This shows $\nu_{l, \infty}^{+}=0$ for every $l \geq 1$.

The case $l=0$ : We have to $\operatorname{show} \operatorname{supp}\left(\nu_{0, \infty}^{+}\right) \subset J_{=0,0}^{+}$. To this end, we consider an arbitrary test function $\phi \in C(Z)$ with

$$
\operatorname{supp}(\phi) \subset\left\{j \in Z \mid \lambda=(j, 0) \in I_{<0}^{+} \cup I_{>0}^{+}\right\}
$$

The outgoing wave condition (3.6) and Proposition 4.3 yield, in the limit $R \rightarrow \infty$,

$$
\sum_{\lambda=(j, 0) \in I_{R} \cap I_{<0}^{+}}\left|\alpha_{\lambda, R}^{+}\right|^{2} \rightarrow 0 \text { and } \sum_{\lambda=(j, 0) \in I_{R} \cap I_{>0}^{+}}\left|\alpha_{\lambda, R}^{+}\right|^{2} P_{\lambda}^{+} \rightarrow 0 .
$$

In the following we assume that $\operatorname{supp}(\phi) \cap\left\{j \in Z \mid \lambda=(j, 0) \in I_{>0}^{+}\right\} \neq \emptyset$, otherwise the proof simplifies. We find

$$
c_{1}:=\inf _{\substack{\lambda=(j, 0) \in I_{>0}^{+} \\ j \in \operatorname{supp}(\phi)}} P_{\lambda}^{+}>0 .
$$

Without loss of generality, we assume $\phi \geq 0$ (otherwise we consider absolute values). For the limit $R \rightarrow \infty$ we calculate

$$
\begin{aligned}
\int_{Z} \phi d \nu_{0, R}^{+} & =\sum_{\substack{\lambda=(j, 0) \in I_{<0}^{+} \cap I_{R} \\
j \in \operatorname{supp}(\phi)}}\left|\alpha_{\lambda, R}^{+}\right|^{2} \phi(j)+\sum_{\substack{\lambda=(j, 0) \in I_{>0}^{+} \cap I_{R} \\
j \in \operatorname{supp}(\phi)}}\left|\alpha_{\lambda, R}^{+}\right|^{2} \phi(j) \\
& \leq\|\phi\|_{\infty} \sum_{\substack{\lambda=(j, 0) \in I_{R} \cap I_{<0}^{+} \\
\mid}}\left|\alpha_{\lambda, R}^{+}\right|^{2}+\left.\|\phi\|_{\infty} \frac{1}{c_{1}} \sum_{\substack{\lambda=(j, 0) \in I_{R} \cap I_{>0}^{+} \\
\mid \alpha_{\lambda, R}^{+}}}\right|^{2} P_{\lambda}^{+} \rightarrow 0 .
\end{aligned}
$$

This shows (4.15) for "+", since $\phi$ with support outside $J_{=0,0}^{+}$was arbitrary. The argument for "-" is analogous.

We next prove that, far away from the interface, solutions to the transmission problem contain only waves that satisfy the frequency condition and the vertical periodicity.

Proposition 4.5 (Bloch measures and frequency condition). Let Assumption 1.1 on $\omega>0$ be satisfied and let $v$ be a solution to the scattering problem (1.1), periodic in vertical direction, satisfying outgoing wave conditions on the left and on the right according to Definition 3.3, with incoming wave $U_{\text {inc }}$ or without incoming wave. Let $\nu_{0, \infty}^{ \pm}$be the Bloch measure to $l=0$ that is generated by $v$. Then

$$
\operatorname{supp}\left(\nu_{0, \infty}^{ \pm}\right) \subset\left\{j \in Z \mid \mu_{0}^{ \pm}(j)=\omega^{2}, j_{2} \in \mathbb{Z} / K\right\} .
$$

Proof. Remark 2.3 implies that the discrete Bloch measures $\nu_{l, R}^{ \pm}$are supported on $\left\{j \in Z \mid j_{2} \in \mathbb{Z} / K\right\}$. This implies that also the limit measure $\nu_{l, \infty}^{ \pm}$are supported on this set. 
In order to show (4.16), it remains to check the frequency condition; we proceed as in the last proof. Let $\phi: Z \rightarrow \mathbb{R}$ be continuous and bounded with $\operatorname{supp}(\phi) \cap\left\{j \mid \mu_{0}(j)=\right.$ $\left.\omega^{2}\right\}=\emptyset$. Arguing with decompositions of the domain of integration, we can consider separately a test-function $\phi \geq 0$ with the property $\phi(j)>0 \Rightarrow \mu_{0}(j)>\omega^{2}$ and a test-function $\tilde{\phi} \geq 0$ with the property $\tilde{\phi}(j)>0 \Rightarrow \mu_{0}(j)<\omega^{2}$. The arguments are analogous and we consider here only $\phi$ as above.

By continuity of $\phi$ we find some $\delta>0$ such that $\mu_{0}(j)-\omega^{2} \geq \delta$ for every $j \in \operatorname{supp}(\phi)$. Our aim is to show that $\int_{Z} \phi d \nu_{0, \infty}^{+}=0$. By definition of the Bloch measure $\nu_{0, \infty}^{+}$we have, as $R \rightarrow \infty$,

$$
\begin{aligned}
0 & \leq \delta \int_{Z} \phi d \nu_{0, \infty}^{+} \leftarrow \delta \int_{Z} \phi d \nu_{0, R}^{+}=\delta \sum_{\lambda=(j, 0) \in I_{R}}\left|\alpha_{\lambda, R}^{+}\right|^{2} \phi(j) \\
& \leq \sum_{\lambda=(j, 0) \in I_{R}}\left(\mu_{0}(j)-\omega^{2}\right)\left|\alpha_{\lambda, R}^{+}\right|^{2} \phi(j) .
\end{aligned}
$$

The result $\int_{Z} \phi d \nu_{0, \infty}^{+}=0$ is shown once we prove that the right hand side of 4.17) vanishes in the limit $R \rightarrow \infty$. In order to show this fact, we recall that the coefficients $\alpha_{\lambda, R}^{+}$are obtained from a Bloch-expansion of the solution at the far right, i.e. $\alpha_{\lambda, R}^{+}=$ $\left\langle u_{R, \eta}^{+}, U_{\lambda}^{+}\right\rangle_{R}$. We calculate

$$
\begin{aligned}
& \sum_{\lambda=(j, 0) \in I_{R}}\left(\mu_{0}(j)-\omega^{2}\right)\left|\alpha_{\lambda, R}^{+}\right|^{2} \phi(j) \\
& \quad \stackrel{(1)}{=} \sum_{\lambda=(j, 0) \in I_{R}} \phi(j) \overline{\alpha_{\lambda, R}^{+}}\left[\left\langle u_{R, \eta}^{+}, \mu_{0}(j) U_{\lambda}^{+}\right\rangle_{R}-\left\langle\omega^{2} u_{R, \eta}^{+}, U_{\lambda}^{+}\right\rangle_{R}\right] \\
& \quad \stackrel{(2)}{=} \sum_{\lambda=(j, 0) \in I_{R}} \phi(j) \overline{\alpha_{\lambda, R}^{+}}\left[\left\langle u_{R, \eta}^{+}, \mathcal{L}_{0} U_{\lambda}^{+}\right\rangle_{R}-\left\langle\omega^{2} u_{R, \eta}^{+}, U_{\lambda}^{+}\right\rangle_{R}\right] \\
& \quad \stackrel{(3)}{=} \sum_{\lambda=(j, 0) \in I_{R}} \phi(j) \overline{\alpha_{\lambda, R}^{+}}\left\langle\mathcal{L}_{0} u_{R, \eta}^{+}-\omega^{2} u_{R, \eta}^{+}, U_{\lambda}^{+}\right\rangle_{R} \\
& \quad \stackrel{(4)}{\leq}\|\phi\|_{\infty}\left(\sum_{\lambda=(j, 0) \in I_{R}}\left|\alpha_{\lambda, R}^{+}\right|^{2}\right)^{1 / 2}\left(\sum_{\lambda=(j, 0) \in I_{R}}\left|\left\langle\mathcal{L}_{0} u_{R, \eta}^{+}-\omega^{2} u_{R, \eta}^{+}, U_{\lambda}^{+}\right\rangle_{R}\right|^{2}\right)^{1 / 2} .
\end{aligned}
$$

In this calculation we used the following: (1) formula for $\alpha_{\lambda, R}^{+}$, (2) the eigenvalue property of $U_{\lambda}$ with eigenvalue $\mu_{\lambda}=\mu_{m}(j)$, (3) integration by parts without boundary terms due to the cut-off function $\eta$, (4) Cauchy-Schwarz inequality. Using orthonormality of the basis functions $U_{\lambda}^{ \pm}$we obtain

$$
\begin{aligned}
& \sum_{\lambda=(j, 0) \in I_{R}}\left(\mu_{0}(j)-\omega^{2}\right)\left|\alpha_{\lambda, R}^{+}\right|^{2} \phi(j) \\
& \quad \leq\|\phi\|_{\infty}\left(f_{W_{R}}\left|\Pi_{m=0}^{\mathrm{ev}} u_{R, \eta}^{+}\right|^{2}\right)^{1 / 2}\left(f_{W_{R}}\left|\Pi_{m=0}^{\mathrm{ev}}\left(\mathcal{L}_{0} u_{R, \eta}^{+}-\omega^{2} u_{R, \eta}^{+}\right)\right|^{2}\right)^{1 / 2} \\
& \quad \leq\|\phi\|_{\infty}\left(f_{W_{R}}\left|u_{R, \eta}^{+}\right|^{2}\right)^{1 / 2}\left(f_{W_{R}}\left|\mathcal{L}_{0} u_{R, \eta}^{+}-\omega^{2} u_{R, \eta}^{+}\right|^{2}\right)^{1 / 2}
\end{aligned}
$$


Since $u_{R, \eta}^{+}$satisfies uniform $L^{2}$-bounds and since $\mathcal{L}_{0} u_{R, \eta}^{+}=\omega^{2} u_{R, \eta}^{+}$holds up to a small $L^{2}$ error, the right hand side of 4.17) is small for large $R>0$. This proves $\int_{Z} \phi d \nu_{0, \infty}^{+}=0$ and hence (4.16) for "+". The proof for "-" is analogous.

Proof of Theorem 1.3. The difference $v$ of two solutions satisfies the outgoing wave condition without an incident wave. Theorem 1.3 is an immediate consequence of Propositions 4.4 and 4.5 .

Proof of Theorem 1.5. We now provide the proof of Theorem 1.5. We therefore assume that: Assumption 1.1 on $\omega>0$ is satisfied and $u$ is a solution of the scattering problem with incoming wave $U_{\text {inc }}$, which has the wave number $k=\left(k_{1}, k_{2}\right)$. In particular, $u$ is a vertically periodic solution of 1.1 such that $u$ and $u-U_{\text {inc }}$ satisfy the outgoing wave conditions on the right and on the left.

Let $\nu_{l, \infty}^{ \pm}$be Bloch measures that are generated by the solution $u$. The frequency condition (1.7) is satisfied and we can therefore use Lemma 3.5. As in Proposition 4.4, case $l \geq 1$, we conclude from (3.15) (and the analogous result for "-") that $\nu_{l, \infty}^{ \pm}=0$ holds for every $l \geq 1$. Moreover, according to Proposition 4.5 we have that $\operatorname{supp}\left(\nu_{0, \infty}^{ \pm}\right) \subset$ $\left\{j \in Z \mid \mu_{0}^{ \pm}(j)=\omega^{2}, j_{2} \in \mathbb{Z} / K\right\}$.

Theorem 1.5 is shown once we verify the following property of the Bloch measure $\nu_{0, \infty}^{ \pm}$

$$
\operatorname{supp}\left(\nu_{0, \infty}^{ \pm}\right) \subset\left\{j \in Z \mid j_{2}=k_{2}\right\} \cup J_{=0,0}^{ \pm} .
$$

Proof of 4.18. We consider the projection $\Pi_{k_{2}}^{\mathrm{vert}} u$ of $u$. This function is again a solution of the scattering problem. Indeed, by Lemma A.2 one has $\Pi_{k_{2}}^{\text {vert } u \in}$ $H_{\text {loc }}^{1}(\mathbb{R} \times(0, h) ; \mathbb{C})$ with periodicity in the $x_{2}$-variable, and for arbitrary test functions $\varphi \in C_{c}^{\infty}(\mathbb{R} \times(0, h))$ there holds

$$
\begin{gathered}
\int_{\mathbb{R}} \int_{0}^{h} \nabla \varphi \cdot a^{\varepsilon} \nabla\left(\Pi_{k_{2}}^{\mathrm{vert}} u\right)=\int_{\mathbb{R}} \int_{0}^{h} \nabla \varphi \cdot a^{\varepsilon} \Pi_{k_{2}}^{\mathrm{vert}}(\nabla u)=\int_{\mathbb{R}} \int_{0}^{h} \Pi_{k_{2}}^{\mathrm{vert}}(\nabla \varphi) \cdot a^{\varepsilon} \nabla u \\
=\int_{\mathbb{R}} \int_{0}^{h} \nabla\left(\Pi_{k_{2}}^{\mathrm{vert}} \varphi\right) \cdot a^{\varepsilon} \nabla u=\omega^{2} \int_{\mathbb{R}} \int_{0}^{h} \Pi_{k_{2}}^{\mathrm{vert}} \varphi u=\omega^{2} \int_{\mathbb{R}} \int_{0}^{h} \varphi \Pi_{k_{2}}^{\mathrm{vert}} u,
\end{gathered}
$$

where we exploited the orthogonality properties of $\Pi_{k_{2}}^{\mathrm{vert}}$ from Lemma A.1 and the solution property of $u$.

As a consequence, the difference $v:=u-\prod_{k_{2}}^{\mathrm{vert}} u$ is a solution of the scattering problem with vanishing incoming wave (just as the difference of two solutions in the proof of Theorem 1.3). The uniqueness statement of Proposition 4.4 implies: Bloch measures (for $l=0$ ) that are generated by $v$ have their support in vertical waves, i.e. in $J_{=0,0}^{ \pm}$.

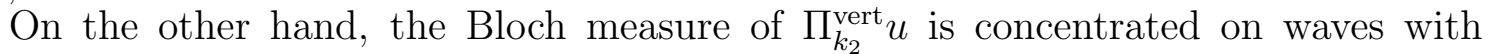
vertical wave number $k_{2}$, i.e. in $\left\{j \in Z \mid j_{2}=k_{2}\right\}$. This follows immediately from the fact that all coefficients $\alpha_{(j, m)}$ with $j_{2} \neq k_{2}$ in the expansion of $\Pi_{k_{2}}^{\mathrm{vert}} u$ vanish.

Since the Bloch measure of $u$ can have its support only in the union of the supports corresponding to $\prod_{k_{2}}^{\mathrm{vert}} u$ and $u-\prod_{k_{2}}^{\mathrm{vert}} u$, the claim (4.18) follows.

Theorem 1.5 is shown. 


\section{Outlook and conclusions}

\section{Remarks on the existence of solutions}

We give some remarks concerning the existence of solutions to the scattering problem. In the end, our radiation condition is "the right one" only if, besides uniqueness, an existence result can be shown.

We formulate the following conjecture: Given a non-singular frequency $\omega>0$, given coefficients $a=a^{\varepsilon}$ that are equal to 1 in the left half plane and $\varepsilon$-periodic in the right half plane, strictly positive and bounded, given finally an incoming wave $U_{\text {inc }}$ as in $(1.2)$ (possibly with a condition on $k$ ), there exists a solution $u$ of the transmission Problem 1.2 .

The idea for an existence proof is the limiting absorption principle (see e.g. [19, 24, 33] for recent contributions): For a positive artificial damping parameter $\delta>0$, we consider the equation

$$
-\nabla \cdot\left((1-i \delta) a(x) \nabla u^{\delta}(x)\right)=\omega^{2} u^{\delta}(x)
$$

for $x \in \Omega=\mathbb{R} \times(0, h)$. Due to the strictly negative imaginary part of the coefficient $(1-i \delta) a(x)$, this equation admits a unique solution $u^{\delta}$ in the Beppo-Levi space $\dot{H}^{1}(\Omega)$ as can be shown with the Lax-Milgram Lemma.

To proceed, two properties must be shown. The first is: The sequence $u^{\delta}$ satisfies estimates in some function space, uniformly in $\delta>0$. Once this is shown, we can consider the distributional limit $u$ of the sequence $u^{\delta}$ as $\delta \rightarrow 0$. As a consequence of distributional convergence, the limit $u$ is a solution of the Helmholtz equation with coefficients $a$.

The intricate part of this approach is to show the second property: The limit $u$ satisfies the outgoing wave condition. We do not see a straightforward argument that yields this condition.

\section{Our outgoing wave condition in a numerical scheme}

The condition of Defitition 3.3 might seem inadequate for numerical purposes on first sight. But it is possible to interpret the condition for a discrete realization: Instead of demanding that limits $R \rightarrow \infty$ vanish, we rather demand that, for some large distance $R$, the left hand side of (3.6) vanishes. In the numerical scheme, this amounts to imposing that a finite number of projections to left-going waves vanishes.

\section{Conclusions}

We have investigated the transmission properties at the boundary of a photonic crystal. Our theorems justify the following: An incoming wave generates, inside the photonic crystal, only those Bloch waves, for which the eigenvalue coincides with the (squared) frequency of the incoming wave. Furthermore, only those Bloch waves can be generated that have the same vertical wave number as the incoming wave; this latter statement is true up to vertical waves. 
Our results rely on a new outgoing wave condition in photonic crystals. The new radiation condition is based on Bloch expansions. It is accompanied by a (weak) uniqueness result, which is expressed with Bloch-measures. The uniqueness result is the basis for the analysis of the transmission problem.

\section{Acknowledgements}

Support of both authors by DFG grant Schw 639/6-1 is gratefully acknowledged.

\section{A Orthogonality and regularity properties}

Lemma A.1 (Orthogonality with periodic weight). Let $f: \mathbb{R} \rightarrow \mathbb{C}$ be $\varepsilon$-periodic and integrable, let $R \in \mathbb{N}$ be an integer.

1. Orthogonality of exponentials. Let $j, \tilde{j} \in Q_{R}$ with $j \neq \tilde{j}$. Then

$$
\int_{0}^{\varepsilon R} f(y) e^{2 \pi i j y / \varepsilon} e^{-2 \pi i \tilde{j} y / \varepsilon} d y=0 .
$$

2. Orthogonality of the vertical pre-Bloch projection. Let $u, v \in L_{\mathrm{loc}}^{2}(\mathbb{R} \times(0, \varepsilon R) ; \mathbb{C})$ and let $k_{2} \in Q_{R}$. Then there holds

$$
\int_{0}^{\varepsilon R} f(y) u\left(x_{1}, y\right) \overline{\Pi_{k_{2}}^{\mathrm{vert}} v\left(x_{1}, y\right)} d y=\int_{0}^{\varepsilon R} f(y) \Pi_{k_{2}}^{\mathrm{vert}} u\left(x_{1}, y\right) \overline{\Pi_{k_{2}}^{\mathrm{vert}} v\left(x_{1}, y\right)} d y .
$$

Proof. 1. By dividing the interval $(0, \varepsilon R)$ into subintervals of length $\varepsilon$, we obtain

$$
\begin{array}{rl}
\int_{0}^{\varepsilon R} & f(y) e^{2 \pi i j y / \varepsilon} e^{-2 \pi i \tilde{j} y / \varepsilon} d y=\sum_{k=0}^{R-1} \int_{k \varepsilon}^{(k+1) \varepsilon} f(y) e^{2 \pi i(j-\tilde{j}) y / \varepsilon} d y \\
= & \sum_{k=0}^{R-1} \int_{0}^{\varepsilon} f(y+k \varepsilon) e^{2 \pi i(j-\tilde{j})(y+k \varepsilon) / \varepsilon} d y=\sum_{k=0}^{R-1} e^{2 \pi i(j-\tilde{j}) k} \int_{0}^{\varepsilon} f(y) e^{2 \pi i(j-\tilde{j}) y / \varepsilon} d y,
\end{array}
$$

where in the last equality we exploited the periodicity of the weight $f$. By setting $C(j, \tilde{j}):=\int_{0}^{\varepsilon} f(y) e^{2 \pi i(j-\tilde{j}) y / \varepsilon} d y$ we conclude

$$
\int_{0}^{\varepsilon R} f(y) e^{2 \pi i j y / \varepsilon} e^{-2 \pi i \tilde{j} y / \varepsilon} d y=C(j, \tilde{j}) \sum_{k=0}^{R-1}\left(e^{2 \pi i(j-\tilde{j})}\right)^{k}=C(j, \tilde{j}) \frac{1-e^{2 \pi i(j-\tilde{j}) R}}{1-e^{2 \pi i(j-\tilde{j})}}=0 .
$$

In the last step we used $j, \tilde{j} \in Q_{R}$, which implies $R(j-\tilde{j}) \in \mathbb{Z}$ and $j, \tilde{j}<1$, and exploited $j \neq \tilde{j}$.

2. Let $u, v$ have vertical pre-Bloch expansions

$$
u\left(x_{1}, x_{2}\right)=\sum_{j_{2} \in Q_{R}} \Phi_{j_{2}}\left(x_{1}, x_{2}\right) e^{2 \pi i j_{2} x_{2} / \varepsilon}, \quad v\left(x_{1}, x_{2}\right)=\sum_{\tilde{j}_{2} \in Q_{R}} \tilde{\Phi}_{\tilde{j}_{2}}\left(x_{1}, x_{2}\right) e^{2 \pi i \tilde{j}_{2} x_{2} / \varepsilon} .
$$


Then the left hand side of $\mathrm{A} .2$ reads

$$
\begin{aligned}
\int_{0}^{\varepsilon R} f(y) u\left(x_{1}, y\right) \overline{\Pi_{k_{2}}^{\text {vert }} v\left(x_{1}, y\right)} d y \\
\quad=\sum_{j_{2} \in Q_{R}} \int_{0}^{\varepsilon R} f(y) \Phi_{j_{2}}\left(x_{1}, y\right) e^{2 \pi i j_{2} y / \varepsilon} \overline{\tilde{\Phi}_{k_{2}}\left(x_{1}, y\right)} e^{-2 \pi i k_{2} y / \varepsilon} d y .
\end{aligned}
$$

Since the function $f(\cdot) \Phi_{j_{2}}\left(x_{1}, \cdot\right) \overline{\tilde{\Phi}_{k_{2}}\left(x_{1}, \cdot\right)}$ is $\varepsilon$-periodic, we can apply the orthogonality (A.1) of Item 1. The sum on the right hand side collapses to $j_{2}=k_{2}$ and we find A.2.

Lemma A.2 (Vertical pre-Bloch projection and gradients). Let $K \in \mathbb{N}, h=\varepsilon K$, and $k_{2} \in Q_{K}$. Let $u \in H_{\mathrm{loc}}^{1}(\mathbb{R} \times(0, h) ; \mathbb{C})$ be periodic in the $x_{2}$-variable. Then the function $\Pi_{k_{2}}^{\text {vert }} u \in H_{\text {loc }}^{1}(\mathbb{R} \times(0, h) ; \mathbb{C})$ is periodic in $x_{2}$ and there holds

$$
\nabla\left(\Pi_{k_{2}}^{\mathrm{vert}} u\right)=\Pi_{k_{2}}^{\mathrm{vert}}(\nabla u)
$$

Proof. Let $u$ have the pre-Bloch expansion $u\left(x_{1}, x_{2}\right)=\sum_{j_{2} \in Q_{K}} \Phi_{j_{2}}\left(x_{1}, x_{2}\right) e^{2 \pi i j_{2} x_{2} / \varepsilon}$. Due to the periodicity of $u$ in the $x_{2}$-variable, each $\Phi_{j_{2}}$ in the above (finite) sum has $H^{1}$-regularity, and thus

$$
\begin{aligned}
\nabla u\left(x_{1}, x_{2}\right) & =\sum_{j_{2} \in Q_{K}} \nabla\left(\Phi_{j_{2}}\left(x_{1}, x_{2}\right) e^{2 \pi i j_{2} x_{2} / \varepsilon}\right) \\
& =\sum_{j_{2} \in Q_{K}}\left[\nabla \Phi_{j_{2}}\left(x_{1}, x_{2}\right)+2 \pi i j_{2} / \varepsilon \Phi_{j_{2}}\left(x_{1}, x_{2}\right) e_{2}\right] e^{2 \pi i j_{2} x_{2} / \varepsilon}
\end{aligned}
$$

where $e_{2}=(0,1) \in \mathbb{R}^{2}$ denotes the second unit vector. Since the expression in the squared brackets is $\varepsilon$-periodic, (A.4) is an expansion of $\nabla u$; uniqueness of the preBloch expansion implies

$$
\begin{aligned}
\Pi_{k_{2}}^{\mathrm{vert}}(\nabla u)\left(x_{1}, x_{2}\right) & =\left(\nabla \Phi_{k_{2}}\left(x_{1}, x_{2}\right)+2 \pi i k_{2} / \varepsilon \Phi_{k_{2}}\left(x_{1}, x_{2}\right) e_{2}\right) e^{2 \pi i k_{2} x_{2} / \varepsilon} \\
& =\nabla\left(\Phi_{k_{2}}\left(x_{1}, x_{2}\right) e^{2 \pi i k_{2} x_{2} / \varepsilon}\right)=\nabla\left(\Pi_{k_{2}}^{\mathrm{vert}} u\right)\left(x_{1}, x_{2}\right)
\end{aligned}
$$

which proves A.3.

Lemma A.3 (Caccioppoli estimate). Let $u \in L_{\text {loc }}^{2}(\mathbb{R} \times(0, h))$ be a vertically periodic solution of the Helmholtz equation $\mathcal{L}_{0} u=\omega^{2} u$. Let $u$ satisfy the uniform $L^{2}$-bounds of Definition 3.3. Then there holds

$$
\frac{1}{R} \int_{W_{R} \backslash W_{R-1}}\left|u_{R}^{ \pm}\right|^{2}+\left|\nabla u_{R}^{ \pm}\right|^{2} \leq C \quad \text { and } \quad f_{W_{R}}\left|u_{R}^{ \pm}\right|^{2}+\left|\nabla u_{R}^{ \pm}\right|^{2} \leq C
$$

with $C$ independent of $R$.

Proof. The proof is, up to translations and a summation, analogous to the proof of the standard Caccioppoli estimate: On a rectangle $(L-1, L+2) \times(0, h)$ we use a cut-off 
function $\theta$ with compact support that depends only on $x_{1}$ and which is identical 1 on $(L, L+1) \times(0, h)$. Testing the equation with $\theta^{2} \bar{u}$ provides

$\int_{L-1}^{L+2} \int_{0}^{h} \omega^{2}|u|^{2} \theta^{2}=\int_{L-1}^{L+2} \int_{0}^{h} \mathcal{L}_{0} u\left(\theta^{2} \bar{u}\right)=\int_{L-1}^{L+2} \int_{0}^{h}\left\{a^{\varepsilon}|\nabla u|^{2} \theta^{2}+2 a^{\varepsilon}(\nabla u \theta) \cdot(\nabla \theta \bar{u})\right\}$.

The Cauchy-Schwarz inquality is used to treat the last term, the first factor is absorbed with Young's inquality in the gradient term, the other consists (up to bounded factors) only of the $L^{2}$-norm of $u$. We conclude that a bound for the $L^{2}$-norm on $(L-1, L+$ $2) \times(0, h)$ implies a bound for the $L^{2}$-norm of the gradient on $(L, L+1) \times(0, h)$. A summation over many squares yields the result.

Lemma A.4 (Regularity of eigenvalue projections $\left.\Pi^{\mathrm{ev}}\right)$. Let $\left(v_{R}\right)_{\mathbb{R} \in \mathbb{N}}$ be a sequence of functions with $H^{2}$-regularity and vanishing boundary data, i.e. $v_{R} \in H_{0}^{2}\left(W_{R} ; \mathbb{C}\right)$. We assume that

$$
f_{W_{R}}\left|v_{R}\right|^{2}+\left|\nabla v_{R}\right|^{2}+\left|\mathcal{L}_{0}\left(v_{R}\right)\right|^{2} \leq C_{0}
$$

holds for $\mathcal{L}_{0}=-\nabla \cdot\left(a^{\varepsilon} \nabla\right)$ with some $R$-independent constant $C_{0}$.

1. Let $\Pi$ be any of the projections of Definition 3.1. Then there exists an $R$ independent constant $C$ such that

$$
f_{W_{R}}\left|\nabla\left(\Pi_{m=0}^{\mathrm{ev}, \pm} v_{R}\right)\right|^{2}+\left|\nabla\left(\Pi_{m \geq 1}^{\mathrm{ev}, \pm} v_{R}\right)\right|^{2}+\left|\nabla\left(\Pi\left(\Pi_{m=0}^{\mathrm{ev}, \pm} v_{R}\right)\right)\right|^{2} \leq C
$$

2. If, additionally, $f_{W_{R}}\left|\Pi_{m \geq 1}^{\mathrm{ev}, \pm} v_{R}\right|^{2} \rightarrow 0$ as $R \rightarrow \infty$, then there holds

$$
f_{W_{R}}\left|\nabla\left(\Pi_{m \geq 1}^{\mathrm{ev}, \pm} v_{R}\right)\right|^{2} \rightarrow 0 \quad \text { as } R \rightarrow \infty
$$

Proof. 1. We omit the superscripts \pm . Concerning (A.7) we note that, because of $\Pi_{m \geq 1}^{\mathrm{ev}} v_{R}=v_{R}-\Pi_{m=0}^{\mathrm{ev}} v_{R}$, the estimate for $\Pi_{m \geq 1}^{\mathrm{ev}} v_{R}$ follows directly from the estimate for $\prod_{m=0}^{\mathrm{ev}} v_{R}$ and Assumption A.6.

Since $\prod_{m=0}^{\mathrm{ev}} v_{R}=\sum_{\lambda=(j, 0) \in I_{R}} \alpha_{\lambda} U_{\lambda}$ is a finite sum of periodic functions, we find that $\Pi_{m=0}^{\mathrm{ev}} v_{R}$ is periodic in $W_{R}$. This allows to calculate, with $0<a_{*} \leq \inf a^{\varepsilon}$,

$$
\begin{aligned}
& a_{*} f_{W_{R}}\left|\nabla\left(\Pi_{m=0}^{\mathrm{ev}} v_{R}\right)\right|^{2} \leq f_{W_{R}} a^{\varepsilon} \nabla\left(\Pi_{m=0}^{\mathrm{ev}} v_{R}\right) \cdot \overline{\nabla\left(\Pi_{m=0}^{\mathrm{ev}} v_{R}\right)} \\
& \quad \stackrel{(1)}{=} f_{W_{R}} \mathcal{L}_{0}\left(\Pi_{m=0}^{\mathrm{ev}} v_{R}\right) \overline{\Pi_{m=0}^{\mathrm{ev}} v_{R}} \stackrel{(2)}{=} f_{W_{R}} \Pi_{m=0}^{\mathrm{ev}}\left(\mathcal{L}_{0} v_{R}\right) \overline{\Pi_{m=0}^{\mathrm{ev}} v_{R}} \\
& \quad \leq\left(f_{W_{R}}\left|\Pi_{m=0}^{\mathrm{ev}}\left(\mathcal{L}_{0} v_{R}\right)\right|^{2}\right)^{1 / 2}\left(f_{W_{R}}\left|\Pi_{m=0}^{\mathrm{ev}} v_{R}\right|^{2}\right)^{1 / 2} \\
& \quad \leq\left(f_{W_{R}}\left|\mathcal{L}_{0} v_{R}\right|^{2}\right)^{1 / 2}\left(f_{W_{R}}\left|v_{R}\right|^{2}\right)^{1 / 2} \leq C_{0} .
\end{aligned}
$$


In (1) we exploited the periodicity of $\prod_{m=0}^{\mathrm{ev}} v_{R}$ to perform integration by parts without boundary terms. In (2), we used the periodicity of $v_{R}$, which yields $\mathcal{L}_{0}\left(\Pi_{m=0}^{\mathrm{ev}} v_{R}\right)=$ $\Pi_{m=0}^{\mathrm{ev}}\left(\mathcal{L}_{0} v_{R}\right)$, as shown in (3.14). In the last line we exploited the norm-boundedness of projections. The claim for $\Pi\left(\Pi_{m=0}^{\mathrm{ev}} v_{R}\right)$ is shown analogously, using again periodicity. This concludes the proof of Relation (A.7).

2. The proof of Relation A.8 is similar and can be interpreted as an interpolation between function spaces. Once more, we exploit that $v_{R}$ has vanishing (and thus periodic) boundary data and that $\prod_{m=0}^{\mathrm{ev}} v_{R}$ is periodic as a finite sum (see Item 1.). Therefore also the difference $\prod_{m \geq 1}^{\mathrm{ev}} v_{R}=v_{R}-\Pi_{m=0}^{\mathrm{ev}} v_{R}$ is periodic. Arguing as above we obtain, as $R \rightarrow \infty$,

$$
a_{*} f_{W_{R}}\left|\nabla\left(\Pi_{m \geq 1}^{\mathrm{ev}} v_{R}\right)\right|^{2} \leq\left(f_{W_{R}}\left|\mathcal{L}_{0} v_{R}\right|^{2}\right)^{1 / 2}\left(f_{W_{R}}\left|\Pi_{m \geq 1}^{\mathrm{ev}} v_{R}\right|^{2}\right)^{1 / 2} \rightarrow 0 .
$$

This shows (A.8) and concludes the proof.

\section{References}

[1] G. Allaire and C. Conca. Bloch wave homogenization and spectral asymptotic analysis. J. Math. Pures Appl. (9), 77(2):153-208, 1998.

[2] G. Allaire, M. Palombaro, and J. Rauch. Diffractive behavior of the wave equation in periodic media: weak convergence analysis. Ann. Mat. Pura Appl. (4), 188(4):561-589, 2009.

[3] G. Allaire, M. Palombaro, and J. Rauch. Diffractive geometric optics for Bloch wave packets. Arch. Ration. Mech. Anal., 202(2):373-426, 2011.

[4] H. Ammari, N. Béreux, and E. Bonnetier. Analysis of the radiation properties of a planar antenna on a photonic crystal substrate. Math. Methods Appl. Sci., 24(13):1021-1042, 2001.

[5] H. Ammari and F. Santosa. Guided waves in a photonic bandgap structure with a line defect. SIAM J. Appl. Math., 64(6):2018-2033, 2004.

[6] A.-S. Bonnet-Ben Dhia, G. Dakhia, C. Hazard, and L. Chorfi. Diffraction by a defect in an open waveguide: a mathematical analysis based on a modal radiation condition. SIAM J. Appl. Math., 70(3):677-693, 2009.

[7] G. Bouchitté and D. Felbacq. Negative refraction in periodic and random photonic crystals. New J. Phys, 7(159, 10.1088), 2005.

[8] G. Bouchitté and B. Schweizer. Homogenization of Maxwell's equations in a split ring geometry. Multiscale Model. Simul., 8(3):717-750, 2010.

[9] C. Castro and E. Zuazua. Une remarque sur l'analyse asymptotique spectrale en homogénéisation. C. R. Acad. Sci. Paris Sér. I Math., 322(11):1043-1047, 1996. 
[10] Y. Chen and R. Lipton. Tunable double negative band structure from non-magnetic coated rods. New Journal of Physics, 12(8):083010, 2010.

[11] D. Colton and R. Kress. Inverse acoustic and electromagnetic scattering theory, volume 93 of Applied Mathematical Sciences. Springer-Verlag, Berlin, second edition, 1998.

[12] A. Efros and A. Pokrovsky. Dielectric photonic crystal as medium with negative electric permittivity and magnetic permeability. Solid State Communications, 129:643-647, 2004.

[13] A. Figotin and A. Klein. Midgap defect modes in dielectric and acoustic media. SIAM J. Appl. Math., 58(6):1748-1773 (electronic), 1998.

[14] S. Fliss. A Dirichlet-to-Neumann approach for the exact computation of guided modes in photonic crystal waveguides. SIAM J. Sci. Comput., 35(2):B438-B461, 2013.

[15] S. Fliss and P. Joly. Wave propagation in locally perturbed periodic media (case with absorption): numerical aspects. J. Comput. Phys., 231(4):1244-1271, 2012.

[16] S. Fliss and P. Joly. Solutions of the time-harmonic wave equation in periodic waveguides: asymptotic behaviour and radiation condition. Arch. Ration. Mech. Anal., 219(1):349-386, 2016.

[17] S. Fliss, D. Klindworth, and K. Schmidt. Robin-to-Robin transparent boundary conditions for the computation of guided modes in photonic crystal wave-guides. BIT, 55(1):81-115, 2015.

[18] H. Helmholtz. Theorie der Luftschwingungen in Röhren mit offenen Enden. J. Reine Angew. Math., 57:1-72, 1860.

[19] V. Hoang. The limiting absorption principle for a periodic semi-infinite waveguide. SIAM J. Appl. Math., 71(3):791-810, 2011.

[20] V. Hoang and M. Radosz. Absence of bound states for waveguides in twodimensional periodic structures. J. Math. Phys., 55(3):033506, 20, 2014.

[21] W. Jäger. Zur Theorie der Schwingungsgleichung mit variablen Koeffizienten in Aussengebieten. Math. Z., 102:62-88, 1967.

[22] J. Joannopoulos, S. Johnson, J. Winn, and R. Meade. Photonic Crystals - Molding the Flow of Light. Princeton University Press, 2008.

[23] P. Joly. An elementary introduction to the construction and the analysis of perfectly matched layers for time domain wave propagation. $S \overrightarrow{\mathrm{e}} M A J ., 57: 5-48,2012$.

[24] P. Joly, J.-R. Li, and S. Fliss. Exact boundary conditions for periodic waveguides containing a local perturbation. Commun. Comput. Phys., 1, 2006. 
[25] A. Lamacz and B. Schweizer. Effective Maxwell equations in a geometry with flat rings of arbitrary shape. SIAM J. Math. Anal., 45(3):1460-1494, 2013.

[26] A. Lamacz and B. Schweizer. A negative index meta-material for Maxwells equations. SIAM J. Math. Anal., to appear, 2017.

[27] C. Luo, S. G. Johnson, J. D. Joannopoulos, and J. B. Pendry. All-angle negative refraction without negative effective index. Phys. Rev. B, 65:201104, May 2002.

[28] R. D. Meade, K. D. Brommer, A. M. Rappe, and J. D. Joannopoulos. Electromagnetic bloch waves at the surface of a photonic crystal. Phys. Rev. B, 44:1096110964, Nov 1991.

[29] E. Moreno, F. J. García-Vidal, and L. Martín-Moreno. Enhanced transmission and beaming of light via photonic crystal surface modes. Phys. Rev. B, 69:121402, Mar 2004 .

[30] S. A. Nazarov. Umov-Mandel'shtam radiation conditions in elastic periodic waveguides. Mat. Sb., 205(7):43-72, 2014.

[31] J. Pendry. Negative refraction makes a perfect lens. Phys. Rev. Lett., 85(3966), 2000 .

[32] A. Pokrovsky and A. Efros. Diffraction theory and focusing of light by a slab of left-handed material. Physica B: Condensed Matter, 338(1-4):333-337, 2003.

[33] M. Radosz. New limiting absorption and limit amplitude principles for periodic operators. Z. Angew. Math. Phys., 66(2):253-275, 2015.

[34] F. Rellich. Über das asymptotische Verhalten der Lösungen von $\Delta u+\lambda u=0$ in unendlichen Gebieten. Jber. Deutsch. Math. Verein., 53:57-65, 1943.

[35] S. H. Schot. Eighty years of Sommerfeld's radiation condition. Historia Math., 19(4):385-401, 1992.

[36] B. Schweizer. The low-frequency spectrum of small Helmholtz resonators. Proc. A., 471(2174):20140339, 18, 2015.

[37] A. Sommerfeld. Die Greensche Funktion der Schwingungsgleichung. Jahresbericht der Deutschen Mathematiker-Vereinigung, 21:309353, 1912. 